\title{
Whole-transcriptome RNA sequencing reveals the global molecular responses and ceRNA regulatory network of mRNAs, IncRNAs, miRNAs and circRNAs in response to copper toxicity in Ziyang Xiangcheng (Citrus junos Sieb. Ex Tanaka)
}

Xing-Zheng $\mathrm{Fu}^{1,2^{*}}$ (D), Xiao-Yong Zhang ${ }^{1,2}$, Jie-Ya Qiu ${ }^{1,2}$, Xue Zhou ${ }^{1,2}$, Meng Yuan ${ }^{1,2}$, Yi-Zhong He $\mathrm{H}^{1,2}$, Chang-Pin Chun ${ }^{1,2}$, Li Cao ${ }^{1,2}$, Li-Li Ling ${ }^{1,2}$ and Liang-Zhi Peng ${ }^{1,2^{*}}$

\begin{abstract}
Background: Copper (Cu) toxicity has become a potential threat for citrus production, but little is known about related mechanisms. This study aims to uncover the global landscape of mRNAs, long non-coding RNAs (IncRNAs), circular RNAs (circRNAs) and microRNAs (miRNAs) in response to Cu toxicity so as to construct a regulatory network of competing endogenous RNAs (ceRNAs) and to provide valuable knowledge pertinent to Cu response in citrus.

Results: Tolerance of four commonly used rootstocks to Cu toxicity was evaluated, and 'Ziyang Xiangcheng' (Citrus junos) was found to be the most tolerant genotype. Then the roots and leaves sampled from 'Ziyang Xiangcheng' with or without $\mathrm{Cu}$ treatment were used for whole-transcriptome sequencing. In total, 5734 and 222 mRNAs, 164 and 5 IncRNAs, 45 and 17 circRNAs, and 147 and 130 miRNAs were identified to be differentially expressed (DE) in Cu-treated roots and leaves, respectively, in comparison with the control. Gene ontology enrichment analysis showed that most of the DEmRNAs and targets of DEInCRNAs and DEmiRNAs were annotated to the categories of 'oxidationreduction', 'phosphorylation', 'membrane', and 'ion binding'. The ceRNA network was then constructed with the predicted pairs of DEmRNAs-DEmiRNAs and DEIncRNAs-DEmiRNAs, which further revealed regulatory roles of these DERNAs in Cu toxicity.
\end{abstract}

Conclusions: A large number of mRNAs, IncRNAs, circRNAs, and miRNAs in 'Ziyang Xiangcheng' were altered in response to $\mathrm{Cu}$ toxicity, which may play crucial roles in mitigation of $\mathrm{Cu}$ toxicity through the ceRNA regulatory network in this Cu-tolerant rootstock.

Keywords: Citrus, Copper, CeRNA, Transcriptome, Non-coding RNA

\footnotetext{
* Correspondence: fuxingzheng@cric.cn; pengliangzhi@cric.cn

${ }^{1}$ Citrus Research Institute, Southwest University, Chongqing 400712, China

Full list of author information is available at the end of the article
}

(c) The Author(s). 2019 Open Access This article is distributed under the terms of the Creative Commons Attribution 4.0 International License (http://creativecommons.org/licenses/by/4.0/), which permits unrestricted use, distribution, and reproduction in any medium, provided you give appropriate credit to the original author(s) and the source, provide a link to the Creative Commons license, and indicate if changes were made. The Creative Commons Public Domain Dedication waiver (http://creativecommons.org/publicdomain/zero/1.0/) applies to the data made available in this article, unless otherwise stated. 


\section{Background}

Copper $(\mathrm{Cu})$ is an essential micronutrient for plant growth and development. As a redox-active transition element, $\mathrm{Cu}$ plays key roles in photosynthesis, respiration, $\mathrm{C}$ and $\mathrm{N}$ metabolism, oxidative stress protection, lignification, pollen fertility, and ethylene perception [14]. Most of the functions rendered by $\mathrm{Cu}$ are ascribed to enzymatically bound $\mathrm{Cu}$, which catalyzes redox reactions [1]. In plants, there are more than $100 \mathrm{Cu}$-containing proteins, such as plastocyanin (PC), copper/zinc superoxide dismutase (CSD), cytochrome c oxidase (COX), laccase (LAC), diamine oxidases (DAO), and polyphenol oxidases [1-3]. Despite being essential, $\mathrm{Cu}$ is easily toxic to plants, even at a supra-optimal level $[5,6]$. Excessive $\mathrm{Cu}$ inhibits plant growth and uptake of other mineral nutrients and alters enzyme systems, membrane integrity and many other biochemical and physiological processes [5]. Unfortunately, in the last decades, $\mathrm{Cu}$ contamination has become a global issue due to the long-term use of $\mathrm{Cu}$-containing fungicides and bactericides, wastewater irrigation, and unconscionable $\mathrm{Cu}$ mining $[6,7]$. In $\mathrm{China}, \mathrm{Cu}$ is ranked as the fourth most contaminating heavy metal of agricultural lands $[7,8]$. Thus, it is important and pressing to gain better understanding of physiological and molecular responses to $\mathrm{Cu}$ excess.

It is worth mentioning that plants have evolutionarily developed a tightly regulated system to balance the uptake, efflux, chelation, distribution, and utilization of $\mathrm{Cu}[9,10]$. In this system, a number of functional proteins (such as $\mathrm{Cu}$ transporters and chaperones), transcription factors (TFs), as well as non-coding RNAs (ncRNAs) may be involved in regulation of $\mathrm{Cu}$ homeostasis. For instance, $\mathrm{Cu}$ transporters, including CTR-like $\mathrm{Cu}$ transporters (COPTs), P-type heavy metal ATPases (HMAs), yellow stripe-like (YSL) proteins, zinc/iron-regulated transporter (ZRT/IRT)-related proteins (ZIPs), and cation diffusion facilitators (CDFs), were shown to directly participate in $\mathrm{Cu}$ uptake, transport, and distribution $[2,3,9,11]$. Among them, COPTs (such as COPT1, COPT2, and COPT6) are mainly responsible for $\mathrm{Cu}$ uptake from soil and redistribution to reproductive organs [12, 13]. The HMAs such as AtHMA5, AtHMA7/RAN1, AtHMA6/PAA1, and AtHMA8/PAA2 of Arabidopsis thaliana are involved in $\mathrm{Cu}$ transport into the xylem, chloroplast, or thylakoid [7, 14-16]. YSL16 protein functions in recycling of $\mathrm{Cu}$ from older tissues to young tissues and grains [17]. Apart from these functional proteins, a conserved transcription factor called SPL7 (Squamosa Promoter binding-Like 7) has been shown to be a central regulator of $\mathrm{Cu}$ homeostasis $[18,19]$. SPL7 regulates the expression of multiple targets, such as COPT1, COPT2, COPT6, cupric reductases FRO4 and FRO5, and Cu-regulated microRNAs (miR397, miR398, miR408 and miR857), that contain reiterative $\mathrm{Cu}$-response elements (CuREs) with a GTAC motif in their promoters under $\mathrm{Cu}$ deficiency or excess [18, 20-22].

In addition to the protein-coding RNAs, emerging evidence has revealed that ncRNAs also play essential regulatory roles in plant responses to abiotic stress [23]. MicroRNAs (miRNAs), a major class of ncRNA with a length of 19 to 24 nucleotides, participate in $\mathrm{Cu}$ homeostasis by repressing translation or directly degrading $\mathrm{Cu}$-related proteins in plants [22, 24]. Previous studies indicate that $\mathrm{Cu}$ deficiency induced the expression of miR397, miR408, and miR857, which repressed a number of $\mathrm{Cu}$-containing proteins, including CSD1, CSD2, LAC, COX subunit 5b (COX5b-1), and $\mathrm{Cu}$ chaperone for SOD (CCS1) [20, 25, 26], while Cu excess downregulated miR398 to induce the expression of CSD1 and CSD2 [27]. Recently, two types of ncRNA, long noncoding RNAs (lncRNAs) and circular RNAs (circRNAs), were discovered in plants. LncRNAs belong to a group of ncRNA longer than 200 nucleotides and can regulate the expression of genes through cis-/trans-acting or miRNA sponges [28, 29]. CircRNAs are endogenous covalently closed circular RNAs that are generated by alternative circularization [30]. A competing endogenous RNA (ceRNA) hypothesis has been proposed that the lncRNAs, circRNAs, mRNAs and pseudogenes can act as ceRNAs to competitively bind common miRNA response elements (MREs), and thus regulate a wide range of biological and developmental processes [31-37]. These ceRNAs are also named miRNA sponges to sequester specific miRNAs and inhibit their functions [30, 31, 34, 35]. In several studies, the ceRNA regulatory theory has been used to uncover molecular mechanisms of plant biology. For example, a ceRNA network was constructed to dissect function of lncRNAs in phosphate starvation of rice [32]. A complex ceRNA network consisting of lncRNAs, mRNAs, and miRNAs was established for maize seed development [33]. A large number of circRNAs, possibly acting as ceRNAs, were found in the ethylene pathway of tomato [38]. Recently, the ceRNA networks were also reported to be related to $A$. thaliana leaf development, tomato flowering, and cucumber heat stress response [35, 37, 39, 40]. However, whether lncRNAs and circRNAs participate in $\mathrm{Cu}$ homeostasis by acting as ceRNAs in plants remains to be investigated.

Citrus is widely grown worldwide. With extensive application of $\mathrm{Cu}$-containing bactericides for controlling citrus canker disease, $\mathrm{Cu}$ toxicity has become a potential threat for citrus. However, relevant researches to understand $\mathrm{Cu}$ toxicity response are greatly limited. In this study, whole-transcriptome RNA sequencing (RNAseq) of Ziyang Xiangcheng (Citrus junos Sieb. ex Tanaka), a widely used citrus rootstock in China, was performed so as to uncover the global molecular responses to $\mathrm{Cu}$ toxicity at both protein-coding RNAs (mRNAs) and 
ncRNAs (lncRNAs, miRNAs, and circRNAs) levels. Moreover, the ceRNA networks were constructed for further revealing the underlying regulatory mechanisms in response to $\mathrm{Cu}$ toxicity.

\section{Results}

\section{Evaluation of tolerance to $\mathrm{Cu}$ toxicity in the citrus} rootstocks

To compare tolerance to $\mathrm{Cu}$ toxicity, four widely used citrus rootstocks, trifoliate orange (TO), 'Ziyang Xiangcheng' (XC), red tangerine (RT), and 'Shatian' pummelo (ST), were subjected to excessive $\mathrm{Cu}$ treatment, followed by evaluation of plant phenotypic and physiological parameters. As shown in Fig. 1, after $25 \mathrm{~d}$ of treatment, top leaves of TO and ST showed a yellow color, while those of XC and RT were almost normal (similar to the phenotype of $\mathrm{CK}$ ). Although the relative increase rate of plant height was significantly suppressed upon $\mathrm{Cu}$ toxicity, XC had a minimal impact among the four rootstocks. Chlorophyll contents were also significantly reduced by $\mathrm{Cu}$ treatment, but $\mathrm{XC}$ had a higher level than the other three rootstocks at 25 and $40 \mathrm{~d}$ of treatment. In addition, excessive $\mathrm{Cu}$ treatment resulted in a drastic increase of MDA in TO, RT, and ST relative to CK, but increase of MDA in $\mathrm{XC}$ was not conspicuous. These results indicated that $\mathrm{XC}$ was the most tolerant genotype to $\mathrm{Cu}$ toxicity among the tested rootstocks. Therefore, $\mathrm{XC}$ was selected for high-throughput RNAseq to reveal global transcriptome of mRNA, lncRNA, circRNA and miRNA in response to $\mathrm{Cu}$ toxicity.

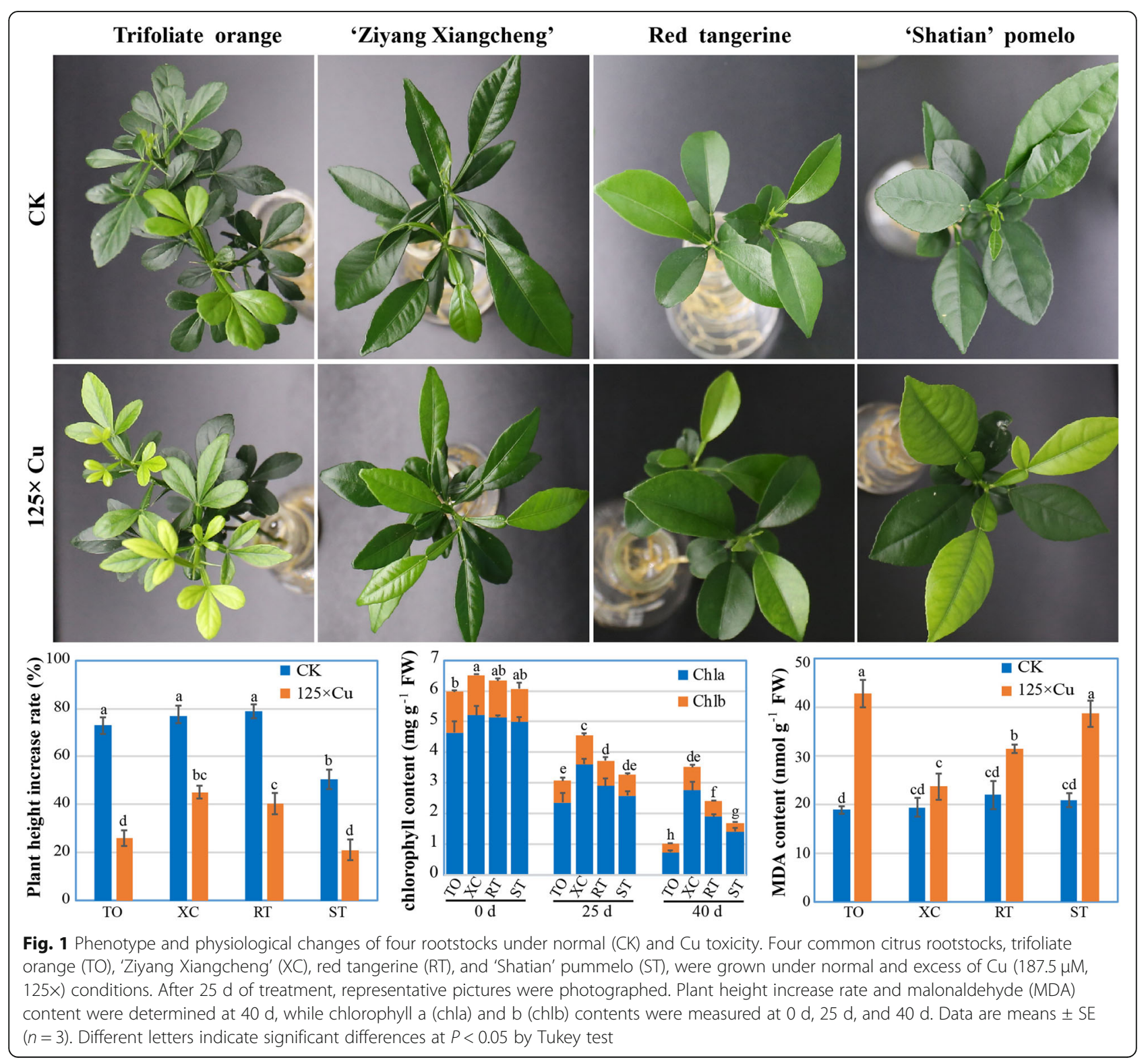




\section{Global responses of mRNA to Cu toxicity}

From RNAseq data, we identified 30,123 protein-coding genes in leaves and roots of $\mathrm{XC}$ by using pummelo as reference genome, and their $\log _{2} \mathrm{FC}$ values are presented as Volcano Plot pictures in Fig. 2a and b, which ranged from -8.2 to 7.9 in roots and from -5.3 to 4.8 in leaves. Among all of these genes, 5734 (2162 up-regulated, 3572 down-regulated) and 222 (132 up-regulated, 90 downregulated) DEmRNAs were identified in $\mathrm{Cu}$-treated root $(\mathrm{CuR} / \mathrm{CKR})$ and leaf $(\mathrm{CuL} / \mathrm{CKL})$ groups, respectively
(Fig. 2c and Additional file 1: Table S1). The number of DEmRNAs in the root was significantly higher than those in the leaf, suggesting that the root had more predominant responses to $\mathrm{Cu}$ toxicity. Moreover, 137 DEmRNAs were common between CuR/CKR and $\mathrm{CuL} /$ $\mathrm{CKL}$, implying that they might participate in the basic response process under $\mathrm{Cu}$ toxicity. A heat map of DEmRNAs showed the general expression profiles of DEmRNAs in each treatment and also showed that the three repeats of each treatment always clustered together

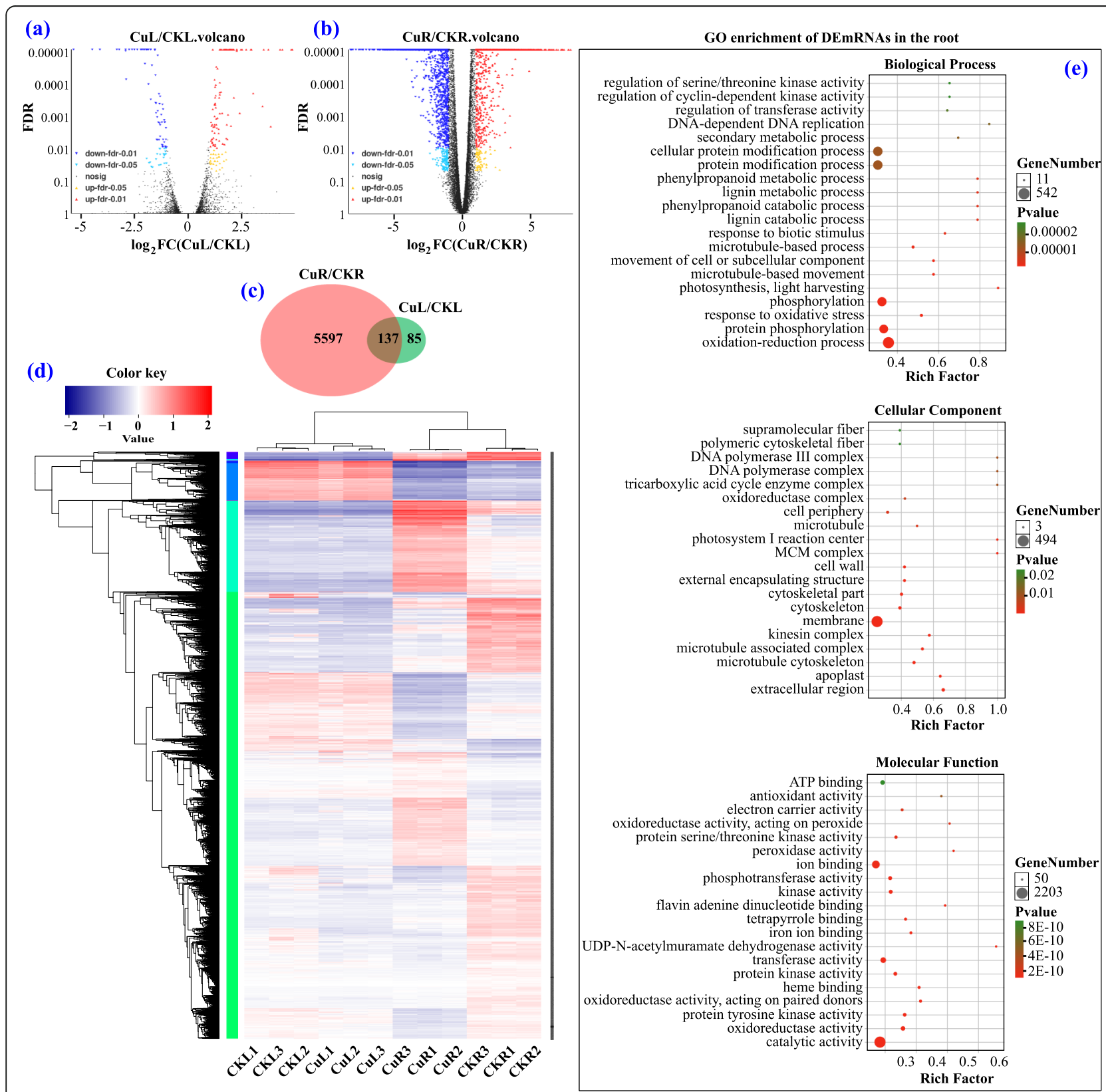

Fig. 2 Identification and analysis of differentially expressed mRNAs (DEmRNAs) under Cu toxicity. $\mathbf{a}, \mathbf{b}$ Volcano Plot pictures showing log 2 FC values and FDR of mRNAs in CUL/CKL and CUR/CKR. $\mathbf{c}$ Venn diagram showing the number of DEmRNAs in CuL/CKL and CUR/CKR. $\mathbf{d}$ Heat map of all DEmRNAs. e GO enrichment of DEmRNAs in the root 
while the $\mathrm{Cu}$-treated group and the $\mathrm{CK}$ group were clustered separately (Fig. 2d).

To explore the functions of the DEmRNAs, GO annotation and GO enrichment analysis were performed. Base on GO annotation, we found that most DEmRNAs in the leaf and root were annotated to the metabolic process, single-organism process, localization process and response to stimulus under biological process (BP), to membrane, cell, organelle, and extracellular region under cellular component (CC), and to binding, catalytic activity, transporter activity, antioxidant activity, transcription factor activity and nutrient reservoir activity under molecular function (MF) (Additional file 10: Figure S1a and b). In particular, there were 317 DEmRNAs in response to stimulus, 596 in the membrane, 45 involved in antioxidant activity, 1 with metallochaperone activity, 32 with molecular transducer activity, 126 nucleic acid binding transcription factors, 18 with nutrient reservoir activity, 19 with receptor activity, and 188 with transporter activity in the root (Additional file 8: Table S8). GO enrichment analysis showed that the oxidation-reduction process, phosphorylation process, photosynthesis process, lignin catabolic process, phenylpropanoid catabolic process, membrane, dehydrogenase activity, peroxidase activity, protein kinase activity, iron ion binding, and heme binding were significantly enriched in the leaf and root (Fig. 2e and Additional file 10: Figure S1c), and these GO terms of DEmRNAs possible have primarily participated in mitigation of $\mathrm{Cu}$ toxicity.

\section{Global responses of IncRNA to $\mathrm{Cu}$ toxicity}

Apart from mRNAs, we also identified 243 known lncRNAs and 1033 novel lncRNAs in XC from the RNAseq data by blasting to known lncRNAs of citrus in the GREENC database and performing CNCI, CPC, CPAT and PfamScan analysis (Fig. 3a). Comparison of the genomic characterizations of the lncRNAs with mRNAs showed that their transcripts were similar in length distribution, except lncRNA had relative higher numbers of long transcripts ( $>4500 \mathrm{bp}$ ) than mRNA; for exon number, a higher percentage of lncRNAs had 2 to 4 exons; in addition, IncRNAs had a shorter ORF length and lower FPKM value (Fig. 3b-e). At a cutoff with an absolute value of $\log _{2} \mathrm{FC}>1$ and $\mathrm{FDR}<0.05,164$ (103 up-regulated, 61 down-regulated) and 5 (1 up-regulated, 4 down-regulated) DElncRNAs were identified in CuR/ $\mathrm{CKR}$ and $\mathrm{CuL} / \mathrm{CKL}$ groups, respectively (Fig. $3 \mathrm{f}$ and Additional file 2: Table S2). The $\log _{2} \mathrm{FC}$ values of DElncRNAs ranged from -10.0 to 13.2 in the root, and -11.1 to 8.8 in the leaf. The general expression profiles of DElncRNAs are shown in Fig. 3g. Similar to DEmRNAs, the DElncRNAs in the Cu-treated group and CK group were clustered separately, while their three repeats were clustered together.
To explore the potential functions of these DElncRNAs, their cis- and trans-targeted mRNAs were predicted with bioinformatics methods (Additional file 3: Table S3). GO annotation of the targets of DElncRNAs in the root showed that they were annotated in 16 terms under BP (mainly involved in metabolic process, cellular process, single-organism process, localization and response to stimulus), 13 terms under CC (mainly involved in membrane, cell, organelle and macromolecular complex), and 10 terms under MF (mainly involved in binding, catalytic activity, transporter activity, electron carrier activity, transcription factor activity, and antioxidant activity) (Additional file 11: Figure S2). GO enrichment analysis of these targets showed that significantly enriched GO terms were the photosynthesis process, phosphorylation process, oxidation-reduction process, lignin catabolic process, phenylpropanoid catabolic process, MCM complex, photosystem I reaction center, extracellular region, membrane, dehydrogenase activity, peroxidase activity, protein kinase activity, iron ion binding, and heme binding (Fig. 3h).

\section{Global responses of circRNA to $\mathrm{Cu}$ toxicity}

In total, 2404 circRNAs were identified in the leaf and root of XC, and $60.48,28.62$, and $10.90 \%$ of them belong to the intergenic region type, exon type, and intron type, respectively (Fig. 4c). The sequence length distribution of circRNAs is shown in Fig. 4a, and most of them were 10, $000 \mathrm{bp}$ to $50,000 \mathrm{bp}$, or shorter than $1200 \mathrm{bp}$. Chromosome 2 (chr2) included maximum circRNAs, followed by chr3, chr5, and chr8 (Fig. 4b). $\log _{2} \mathrm{FC}$ values of circRNAs are displayed in Fig. 4d and e, and 45 (28 up-regulated, 17 down-regulated) and 17 (11 up-regulated, 6 downregulated) DEcircRNAs were identified in CuR/CKR and $\mathrm{CuL} / \mathrm{CKL}$ groups, respectively (Fig. $4 \mathrm{f}$ and Additional file 4: Table S4), among which, 1 DEcircRNAs were common between $\mathrm{CuR} / \mathrm{CKR}$ and $\mathrm{CuL} / \mathrm{CKL}$. A heat map showed the general expression profiles of DEcircRNAs in each treatment, and most DEcircRNAs were highly expressed in the $\mathrm{CuR}$ group (Fig. 4g). These results suggest that the circRNAs are also involved in the responses to $\mathrm{Cu}$ toxicity.

\section{Global responses of miRNA to Cu toxicity}

In the present study, a total of 23,333,512, 24,526,156, $21,822,295$, and $25,871,923$ raw reads were generated from CKL, CKR, CuL and CuR small RNA libraries, respectively. Of these raw reads, we obtained 15,050,388, $15,173,260,13,474,928$ and 13,748,074 clean reads after removing adaptor sequences, low-quality sequences, and reads shorter than $18 \mathrm{nt}$ and longer than $32 \mathrm{nt}$. The lengths of most clean reads were 20-24 nt (Fig. 5a). Small RNA classification showed that $81 \%$ of clean reads were rRNA (42\%) and unmatched (39\%), and there were also $14 \%$ miRNA, $5 \%$ tRNA, and $1 \%$ of other types (Fig. 


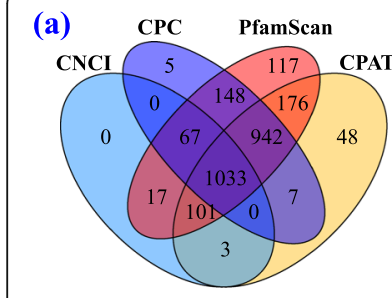

(c) IncRNA vs mRNA on exon number

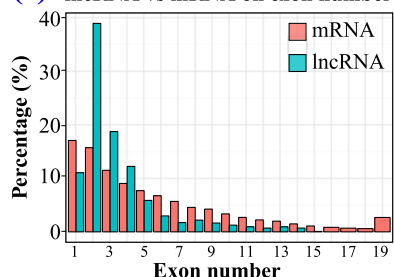

(e) IncRNA vs mRNA on FPKM value

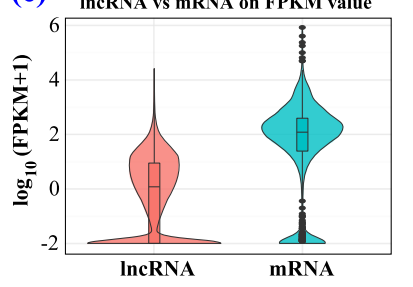

(f)

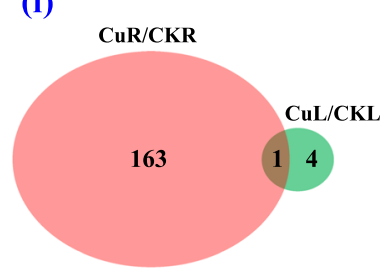

(b) IncRNA vs mRNA on length

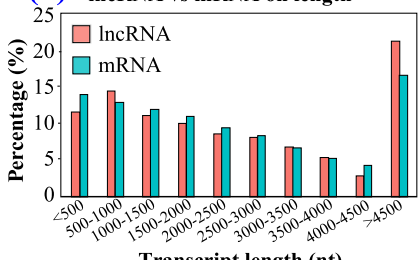

Transcript length (nt)

(d) IncRNA vs mRNA on ORF length

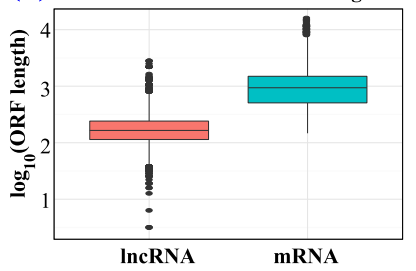

$\mathrm{CuR} / \mathrm{CKR}$

(g)
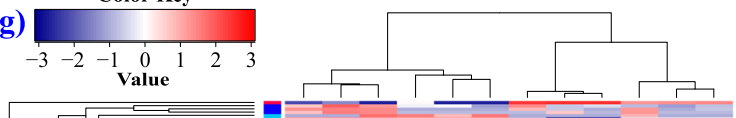
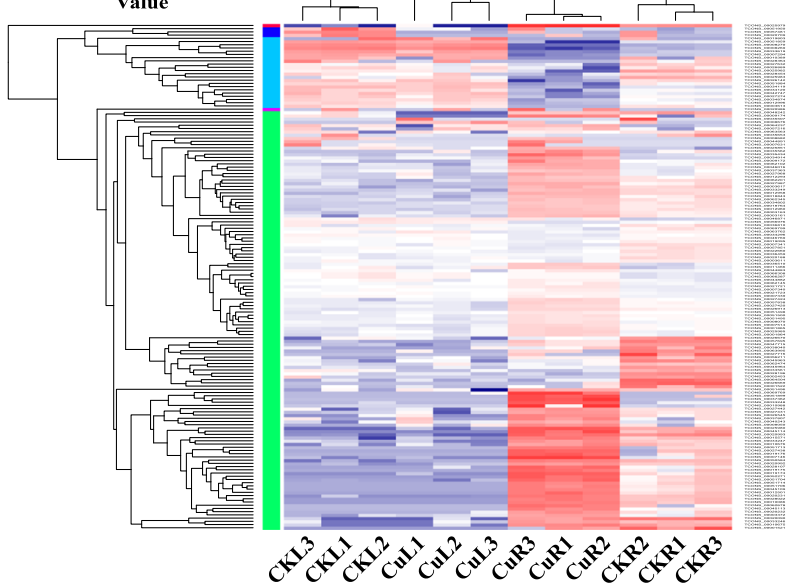

protein kinase activity protein tyrosine kinase activity

oxidoreductase activity, acting on paired donors oxidoreductase activity
catalytic activity

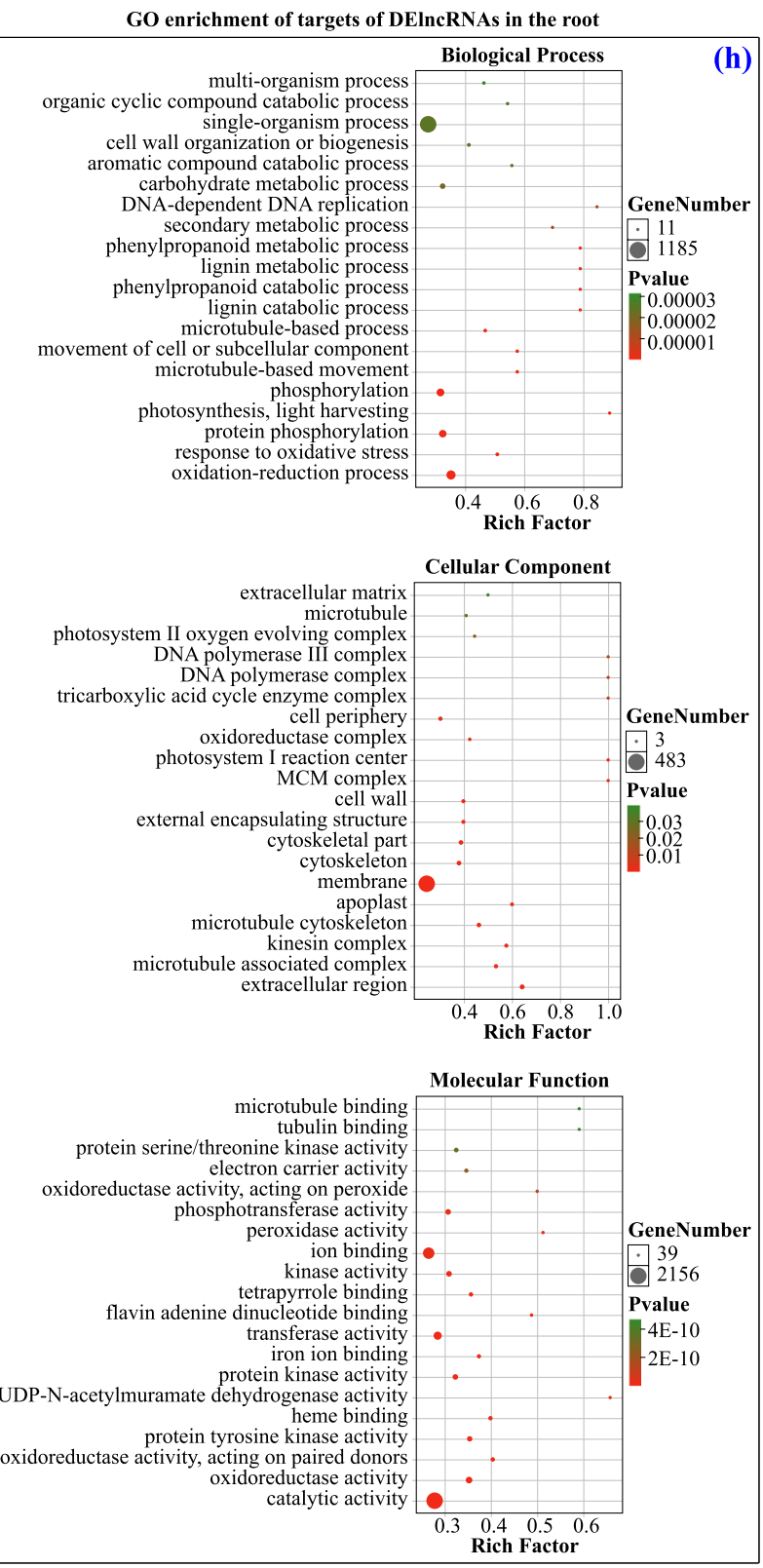

Fig. 3 Identification and analysis of differentially expressed IncRNAs (DEIncRNAs) under Cu toxicity. a Venn diagram showing the number of IncRNAs identified by CNCl, CPC, PfamScan and CPAT methods. b-e Comparison of IncRNA with mRNA with respect to the transcript length, exon number, ORF length and FPKM value. $\mathbf{f}$ Venn diagram showing the number of DElncRNAs in CuL/CKL and CuR/CKR. $\mathbf{g}$ Heat map of all DEIncRNAs. $\mathbf{h}$ GO enrichment of targets of DEIncRNAs in the root

5b). From $14 \%$ of clean reads, we finally identified 149 known miRNAs and 336 novel miRNAs. The top 10 expressed miRNAs in each sample are shown in Fig. $5 \mathrm{c}$ and $\mathrm{d}$, and miR166a-3p and nov-m0105-3p exhibited the highest expression abundance. From known miRNAs 12 (10 up-regulated, 2 down-regulated) and 3 (all up-regulated) DEmiRNAs, and from novel miRNAs 135 (26 up-regulated, 109 down-regulated) and 127 (42 up-regulated, 85 downregulated) DEmiRNAs were identified in CuR/CKR and $\mathrm{CuL} / \mathrm{CKL}$ groups, respectively (Fig. $5 \mathrm{e}$ and $\mathrm{f}$ and Additional file 5: Table S5). The general expression profiles of these DEmiRNAs are shown in Fig. $5 \mathrm{~g}$ and $\mathrm{h}$. Their expression levels exhibited obvious differences between $\mathrm{CK}$ and $\mathrm{Cu}$-treated samples and between root and leaf samples.

Targeted mRNAs of these DEmiRNAs are listed in Additional file 6: Table S6. We found that $84.7 \%$ of DEmRNAs in the leaf $(188 / 222)$ and $81.0 \%$ of DEmRNAs 


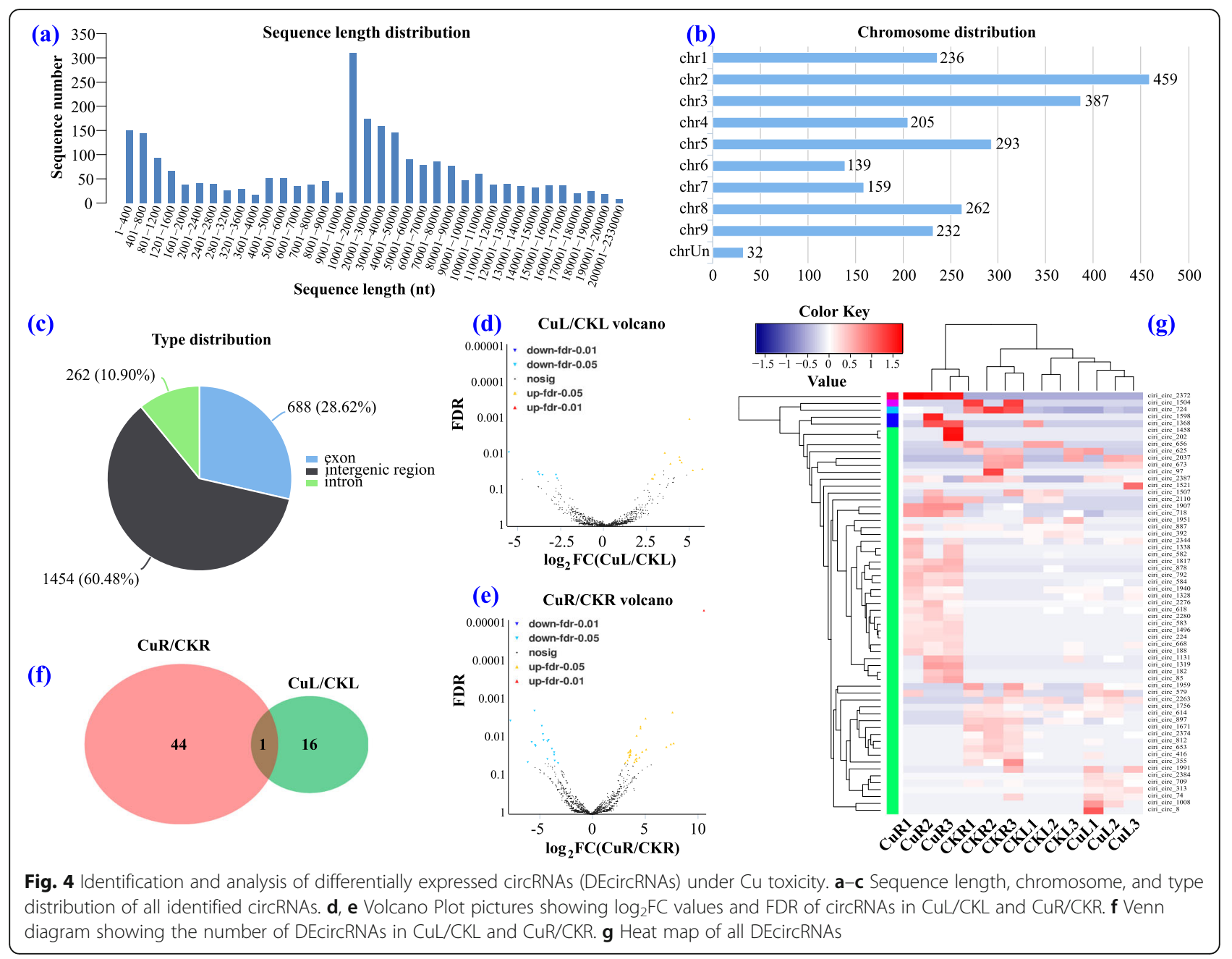

in the root (4642/5734) were targeted by one or multiple DEmiRNAs. GO annotation of the targets of DEmiRNAs in the root showed that most of them were annotated to the metabolic process, cellular process, single-organism process, localization process, and response to stimulus under BP, to membrane, cell, organelle and macromolecular complex under $\mathrm{CC}$, and to binding, catalytic activity and transporter activity under MF (Additional file 12: Figure S3a and b). GO enrichment analysis showed that the significantly enriched GO terms of the targets of known DEmiRNAs in root were photosynthesis, microtubulebased movement, carbohydrate metabolic process, DNA polymerase complex, microtubule, membrane, cellulose synthase activity, microtubule binding, and catalytic activity, while those of novel DEmiRNAs were microtubule-based movement process, phosphorylation process, protein modification process, oxidationreduction process, DNA polymerase complex, kinesin complex, extracellular region, membrane, protein kinase activity, transferase activity, anion binding, and catalytic activity (Fig. 6a and b).

\section{CeRNA regulatory network in response to $\mathrm{Cu}$ toxicity}

To reveal the global regulatory network of proteincoding RNAs and ncRNAs under $\mathrm{Cu}$ toxicity, a ceRNA network was constructed using DEmiRNAs, DEmRNAs, DElncRNAs, and DEcircRNAs based on ceRNA theory. In total, 5739 DEmRNAs, 64 DElncRNAs, and 5 DEcircRNAs were predicted as targets of 251 miRNAs in the root and leaf. When their correlation was further filtered with $\mathrm{SCC}<-0.5$, we obtained 3819 DEmiRNA-DEmRNA and 10 DEmiRNADElncRNA interactions in the root and 12 DEmiRNA-DEmRNA interactions in the leaf (Fig. 7). In this ceRNA network, Nov-m0238-3p, Nov-m00745p, Nov-m0183-3p, miR166c-5p, Nov-m0128-3p, Novm0328-5p, miR165a-5p, and miR535c are involved in more than 100 nodes, suggesting that they may act as core regulators. In addition, lncRNAs including TCONS_00012501, TCONS_00012960, TCONS_00025983, TCONS_00027274, TCONS_00034874, TCONS_00036810, TCONS_00042747, TCONS_00051884 and TCONS_ 00062474 participated in the network. 

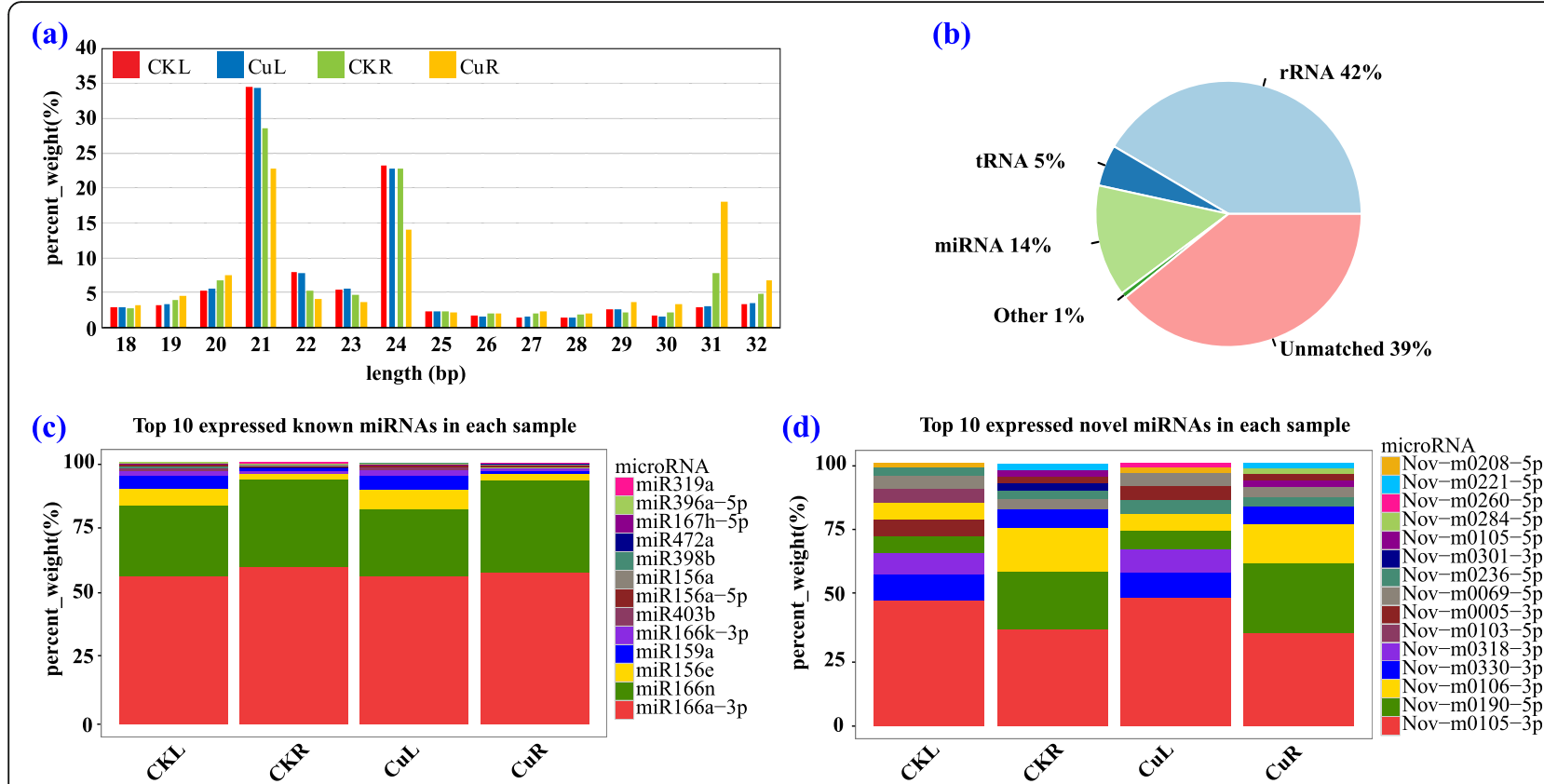

(e)

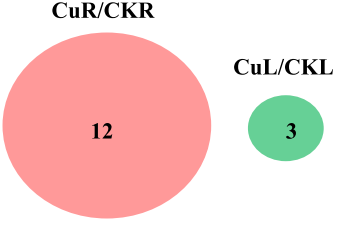

(f)

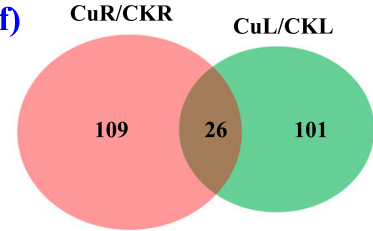

(g)

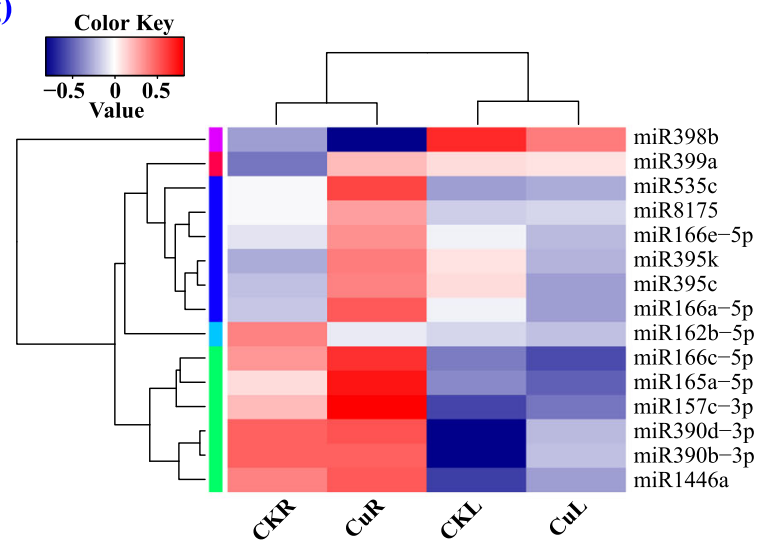

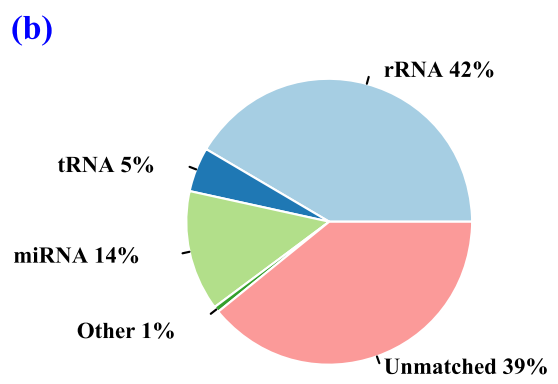

(b) (h)

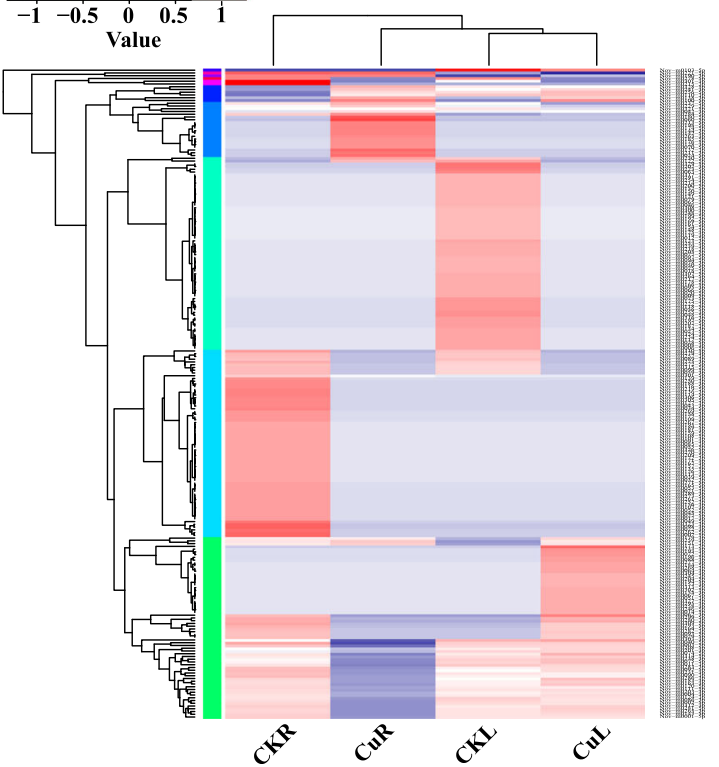

Fig. 5 Identification and analysis of differentially expressed miRNAs (DEmiRNAs) under Cu toxicity. a Length distribution of all identified small RNAs. $\mathbf{b}$ Percentage of different types of small RNAs. $\mathbf{c}$, $\mathbf{d}$ Top 10 expressed known and novel miRNAs in each sample. e, $\mathbf{f}$ Venn diagram showing the number of known (e) and novel (f) DEmiRNAs in CuL/CKL and CUR/CKR. $\mathbf{g}$, $\mathbf{h}$ Heat map of all known (g) and novel (h) DEmiRNAs

Considering that the network contains enormous information and each one cannot be displayed in the figure, we constructed a mini-ceRNA network by reducing the mRNA amount. We identified 284 known $\mathrm{Cu}-$ related mRNAs that were reported directly or indirectly in previous literatures from all DEmRNAs of the root; they included $\mathrm{Cu}$-related regulators (SPL, YSL, CDPK, MAPK, and SUMO E3 Ligase, etc.), transporters (COPT,
HMA, PPA, CDF, ZIP, OPT, and ABC transporter, etc.) and enzymes (LAC, CSD, CCS, PC, and COX, etc.) according to their functional description (Additional file 7 : Table S7 and Additional file 8: Table S8). The miniceRNA network was constructed with these 284 DEmRNAs and all DEmiRNAs, DElncRNAs, and DEcircRNAs. Finally, only 261 DEmiRNA-DEmRNA and 10 DEmiRNADElncRNA interactions $\quad(\mathrm{SCC}<-0.5) \quad$ containing 18 


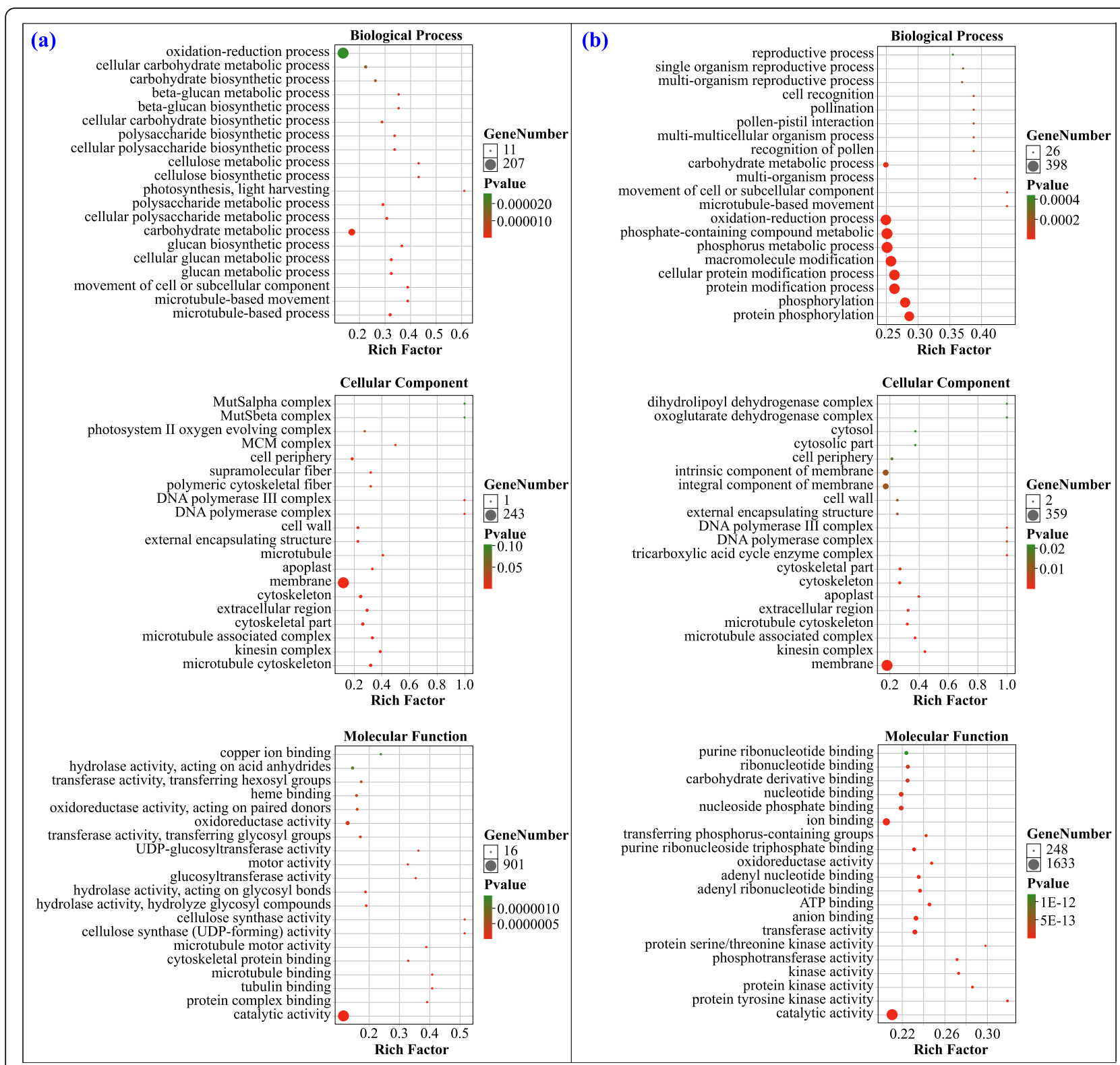

Fig. $6 \mathrm{GO}$ enrichment of targets of known (a) and novel (b) DEmiRNAs in the root

DEmiRNAs, 129 DEmRNAs, and 9 DElncRNAs were obtained and are displayed in Fig. 8. DEmiRNAs including miR166a-5p, miR395c, miR535c, miR395k, miR166c-5p, miR165a-5p, and miR399a were involved in more than 20 nodes, and all of them were up-regulated in the $\mathrm{CuR}$. A known Cu-related key miRNA, miR398b, was identified in the network, which down-regulated and interacted with 5 DEmRNAs and 2 DElncRNAs. In addition, many known Cu-related key mRNAs such as SPL (Cg5g011720, Cg6g012520, Cg7g016770), YSL (Cg7g013630), HMA (Cg5g002920, Cg5g002930, Cg4g021370), ABC transporter (Cg5g018290, Cg5g027620, Cg3g011050, Cg3g009290, and Cg5g021160 etc.), LAC (Cg6g004840, Cg7g002640,
Cg6g004880) and ZIP (Cg8g019240, Cg9g029160, Cg2g037280) were down-regulated in the network.

\section{qRT-PCR validated expression correlation between miRNAs and ceRNAs under $\mathrm{Cu}$ toxicity}

To confirm the results of RNAseq and validate expression correlation of miRNA and their targets, six miRNAs (miR398b, miR8175, miR157c-3p, miR166a-5p, miR165a5p, and Nov-m0284-5p) and their targeted mRNAs and IncRNAs were selected from the ceRNA network for qRT-PCR. As shown in Table 1, the qRT-PCR results agreed well with RNAseq data except several lowabundance mRNAs were undetectable. In addition, the 


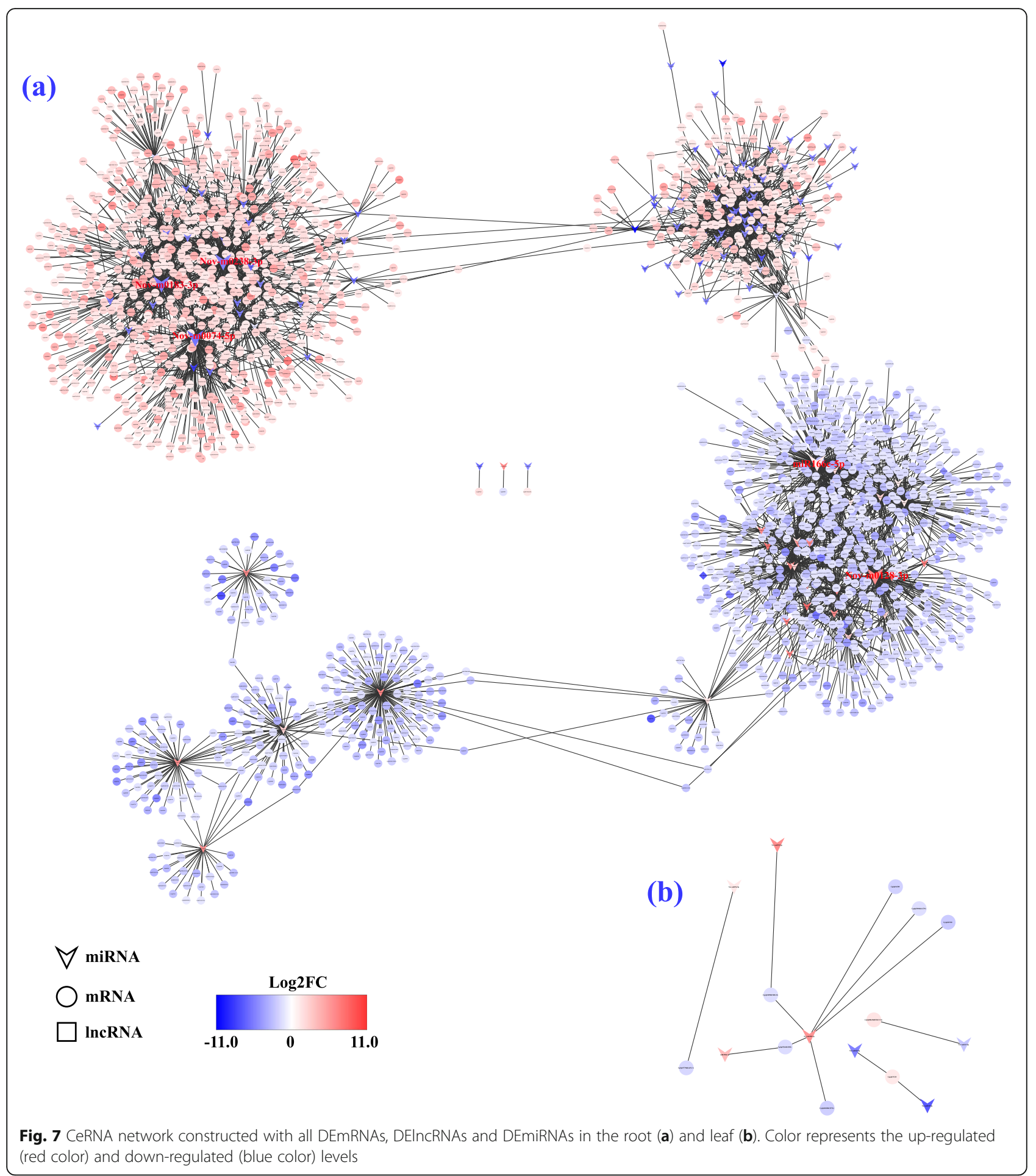

miRNA and its targets showed quite correct up- or down-regulated relationships. For example, miR398 was down-regulated and all of its predicted targets were upregulated; miR8175 was up-regulated and all of its targets were down-regulated. This result not only suggests reliability of RNAseq data in this study, but also validates the negatively correlated expression between miRNAs and ceRNAs.

\section{Expression patterns of candidate RNAs between XC and TO}

To further understand the $\mathrm{Cu}$ tolerance mechanism of $\mathrm{XC}$, comparative analysis of the expression of known $\mathrm{Cu}$-related miRNAs and ceRNAs was performed between $\mathrm{Cu}$-tolerant $\mathrm{XC}$ and $\mathrm{Cu}$-sensitive TO. As shown in Fig. 9, miR398b and its targets (Cg1g001620, 


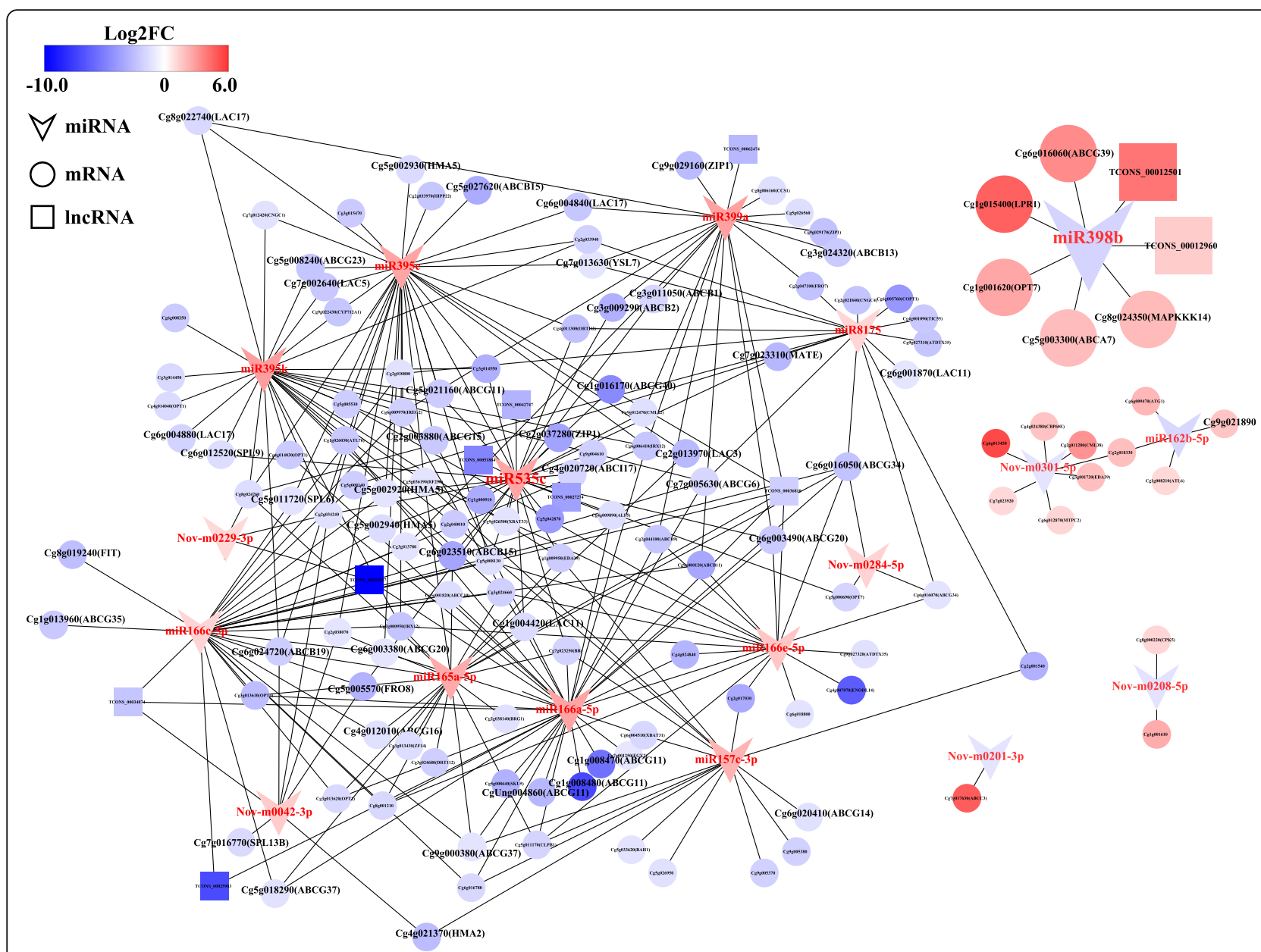

Fig. 8 Mini-ceRNA network constructed with 284 known Cu-related DEmRNAs and all DElncRNAs and DEmiRNAs in the root. Color represents the up-regulated (red color) and down-regulated (blue color) levels

Cg1g015400, Cg5g003300 and TCONS_0012960) were down-regulated or up-regulated in both roots of XC and $\mathrm{TO}$ at $1 \mathrm{~d}, 3 \mathrm{~d}$ and $5 \mathrm{~d}$ of $\mathrm{Cu}$ toxicity. However, the absolute values of $\log _{2} \mathrm{FC}$ in $\mathrm{XC}$ were significantly higher than those in $\mathrm{TO}$ at most time points. The similar changes were found for miR157c-3P and miR535c, as well as their targeted mRNAs and lncRNAs. These results suggest that the $\mathrm{Cu}$-tolerant $\mathrm{XC}$ had occurred much more drastic expression of the $\mathrm{Cu}$-related miRNAs, mRNAs and lncRNAs under $\mathrm{Cu}$ toxicity, which may be the important reason that leads to higher $\mathrm{Cu}$ tolerance in $\mathrm{XC}$.

\section{Discussion}

The ceRNA hypothesis is now widely accepted since it was reported several years ago [31]. Despite great progress in understanding human disease using the ceRNA theory [41], relatively fewer studies have been carried out in plants. In the ceRNA network, miRNAs play critical role in connection and regulation of different
ceRNAs, such as mRNAs, lncRNAs and circRNAs. The mRNAs can be transcribed into proteins that exhibit direct functions, whereas the lncRNAs and circRNAs, not transcribed, can indirectly influence the expression of mRNAs by competitively binding the common miRNAs [31]. Based on this theory, we try to construct a ceRNA regulatory network so as to elucidate the mechanisms underlying tolerance to excessive $\mathrm{Cu}$ in the tolerant genotype.

In this study, $96.3 \%$ of DEmRNAs were predicted as the targets of 251 DEmiRNAs, which constitute 3819 DEmiRNA-DEmRNA interactions in the root and 12 in the leaf. This result suggests that most of the DEmRNAs may be regulated by the miRNAs under $\mathrm{Cu}$ toxicity. From the constructed ceRNA network, we found several miRNAs, including substantially down-regulated miR398b and up-regulated miR165a-5p, miR166a-5p, miR166c-5p, miR395c, miR395k, miR399a and miR535c, which have been previously reported to play a role in metal stress response. Among them, miR398 was shown to be 
Table 1 Confirmation of expression levels of miRNAs and their targeted IncRNAs and mRNAs based on qRT-PCR

\begin{tabular}{|c|c|c|c|c|}
\hline & RNA & $\begin{array}{l}\log _{2} F C(C u R / C K R) \\
\text { by RNAseq }\end{array}$ & $\begin{array}{l}\log _{2} F C(C u R / C K R) \\
\text { by qPCR }\end{array}$ & A. thaliana description \\
\hline & miR398b & -1.72561 & -1.22962 & \\
\hline \multirow[t]{8}{*}{ predicted targets of miR398b } & $\begin{array}{l}\text { TCONS } \\
00012501\end{array}$ & 3.6 & 4.77311 & \\
\hline & $\begin{array}{l}\text { TCONS } \\
00012960\end{array}$ & 1.32 & 1.392306 & \\
\hline & Cg6g016060 & 3.06 & 3.721868 & ABC transporter $\mathrm{G}$ family member 39 \\
\hline & Cg1g001620 & 2.35 & 2.21382 & Oligopeptide transporter 7 \\
\hline & Cg1g015400 & 4.19 & 3.447331 & Multicopper oxidase LPR1 \\
\hline & Cg5g003300 & 1.76 & 1.164213 & ABC transporter A family member 7 \\
\hline & Cg8g024350 & 1.82 & undetected & Mitogen-activated protein kinase kinase 14 \\
\hline & miR8175 & 1.089447 & 1.058019 & \\
\hline \multirow[t]{10}{*}{ predicted targets of miR8175 } & Cg6g001870 & -1.02 & undetected & Laccase-11 \\
\hline & Cg4g001090 & -2.13 & -1.88539 & Protein TIC 55, chloroplastic \\
\hline & Cg9g027310 & -2.25 & -2.70294 & Detoxifying efflux carrier 35 \\
\hline & Cg2g021840 & -2.35 & -4.28181 & Cyclic nucleotide-gated ion channel 4 \\
\hline & Cg6g016050 & -2.39 & -1.89089 & ABC transporter $\mathrm{G}$ family member 34 \\
\hline & Cg2g047100 & -2.4 & -2.92599 & Ferric reduction oxidase 7 \\
\hline & Cg7g023310 & -2.83 & -2.96635 & MATE efflux family protein 1 \\
\hline & Cg2g001540 & -3.09 & -4.80108 & Transducin/WD40 repeat-like superfamily protein \\
\hline & Cg6g005760 & -4.11 & -4.6826 & Copper transporter 1 \\
\hline & miR157c-3p & 1.98457 & 1.730227 & \\
\hline \multirow[t]{14}{*}{ predicted targets of miR157c-3p } & Cg5g033620 & -1.1 & -2.39108 & E3 ubiquitin-protein ligase $\mathrm{BAH} 1$ \\
\hline & Cg6g020410 & -1.21 & -1.88539 & ABC transporter G family member 14 \\
\hline & Cg5g026950 & -1.23 & undetected & RING/U-box superfamily protein \\
\hline & Cg6g016780 & -1.54 & -2.09326 & RCC1family with FYVE zinc finger domain \\
\hline & Cg5g011170 & -1.72 & -1.56999 & Chaperone protein $\mathrm{ClpB1}$ \\
\hline & Cg7g023250 & -1.76 & -1.79581 & E3 ubiquitin ligase BIG BROTHER \\
\hline & Cg9g005380 & -1.81 & -1.71421 & Copper amine oxidase family protein \\
\hline & Cg9g005370 & -1.83 & -1.90385 & Copper amine oxidase family protein \\
\hline & Cg1g009950 & -2.04 & -3.01473 & calmodulin-binding family protein \\
\hline & Cg4g021370 & -2.65 & -3.17684 & Cadmium/zinc-transporting ATPase HMA2 \\
\hline & Cg2g001540 & -3.09 & -4.80108 & Transducin/WD40 repeat-like superfamily protein \\
\hline & Cg2g017030 & -3.4 & -4.59911 & Transducin/WD40 repeat-like superfamily protein \\
\hline & Cg6g016070 & -1.13 & -1.13584 & ABC transporter G family member 34 \\
\hline & Nov-m0284-5p & 1.198039 & 1.01773 & \\
\hline \multirow{4}{*}{$\begin{array}{l}\text { predicted targets of Nov-m0284- } \\
5 p\end{array}$} & Cg6g016070 & -1.13 & -1.13584 & ABC transporter G family member 34 \\
\hline & Cg5g000690 & -1.7 & undetected & Oligopeptide transporter 7 \\
\hline & Cg6g016050 & -2.39 & -1.89089 & ABC transporter G family member 34 \\
\hline & miR166a-5p & 2.37994 & 2.444839 & \\
\hline \multirow[t]{3}{*}{ predicted targets of miR166a-5p } & $\begin{array}{l}\text { TCONS_} \\
00025983\end{array}$ & -7.16 & -3.31462 & \\
\hline & $\begin{array}{l}\text { TCONS_} \\
00034874\end{array}$ & -2.3 & -2.30161 & \\
\hline & $\begin{array}{l}\text { TCONS } \\
00036810\end{array}$ & -1.92 & -1.70539 & \\
\hline
\end{tabular}


Table 1 Confirmation of expression levels of miRNAs and their targeted IncRNAs and mRNAs based on qRT-PCR (Continued)

\begin{tabular}{|c|c|c|c|c|}
\hline & RNA & $\begin{array}{l}\log _{2} \mathrm{FC}(\mathrm{CuR} / \mathrm{CKR}) \\
\text { by RNAseq }\end{array}$ & $\begin{array}{l}\log _{2} \mathrm{FC}(\mathrm{CUR} / \mathrm{CKR}) \\
\text { by } \mathrm{qPCR}\end{array}$ & A. thaliana description \\
\hline & Cg2g034240 & -1.17 & -2.05887 & MATE efflux family protein \\
\hline & Cg3g013610 & -2.61 & -3.0194 & Oligopeptide transporter 4 \\
\hline & Cg3g013620 & -1.5 & -1.66687 & Oligopeptide transporter 2 \\
\hline & Cg5g001820 & -1.35 & undetected & $\mathrm{ABC}$ transporter $\mathrm{C}$ family member 10 \\
\hline & $\mathrm{Cg} 5 \mathrm{~g} 008640$ & -3.08 & -3.69684 & Monocopper oxidase-like protein SKU5 \\
\hline & $\mathrm{Cg} 5 \mathrm{~g} 011170$ & -1.72 & -1.56999 & Chaperone protein ClpB1 \\
\hline & $\mathrm{Cg} 5 \mathrm{~g} 018290$ & -1.11 & -0.16429 & ABC transporter $\mathrm{G}$ family member 37 \\
\hline & Cg6g006410 & -2.24 & -3.65275 & Laccase-4 \\
\hline & Cg6g016050 & -2.39 & -1.89089 & ABC transporter $\mathrm{G}$ family member 34 \\
\hline & Cg6g016070 & -1.13 & -1.13584 & ABC transporter $\mathrm{G}$ family member 34 \\
\hline & Cg7g005630 & -1.6 & -1.39821 & ABC transporter $\mathrm{G}$ family member 6 \\
\hline & Cg7g023250 & -1.76 & -1.79581 & E3 ubiquitin ligase BIG BROTHER \\
\hline & Cg8g001210 & -1.53 & undetected & Major facilitator superfamily protein \\
\hline & Cg9g000380 & -1.08 & undetected & ABC transporter $\mathrm{G}$ family member 37 \\
\hline & $\operatorname{miR} 165 a-5 p$ & 2.203897 & 2.322514 & \\
\hline \multirow[t]{15}{*}{ predicted targets of miR165a-5p } & $\begin{array}{l}\text { TCONS_ } \\
00036810\end{array}$ & -1.92 & -1.70539 & \\
\hline & Cg2g013970 & -2.75 & -2.55165 & Laccase-3 \\
\hline & Cg3g013430 & -1.32 & -1.66301 & MATE efflux family protein \\
\hline & Cg3g013610 & -2.61 & -3.01937 & Oligopeptide transporter 4 \\
\hline & Cg3g013620 & -1.5 & -1.66687 & Oligopeptide transporter 2 \\
\hline & Cg3g024660 & -1.87 & -2.19929 & Major facilitator superfamily protein \\
\hline & Cg3g024680 & -1.96 & -2.62043 & Plastocyanin major isoform, chloroplastic \\
\hline & $\mathrm{Cg} 5 g 011170$ & -1.72 & -1.56999 & Chaperone protein ClpB1 \\
\hline & $\mathrm{Cg} 5 \mathrm{~g} 018290$ & -1.11 & -0.16429 & ABC transporter $\mathrm{G}$ family member 37 \\
\hline & Cg6g016050 & -2.39 & -1.89089 & ABC transporter $\mathrm{G}$ family member 34 \\
\hline & Cg7g000950 & -2.35 & -2.44466 & Laccase-4 \\
\hline & Cg7g023250 & -1.76 & -1.79581 & E3 ubiquitin ligase BIG BROTHER \\
\hline & Cg8g001210 & -1.53 & undetected & Major facilitator superfamily protein \\
\hline & Cg8g024760 & -1.34 & -0.91518 & $\begin{array}{l}\text { Calcineurin-like metallo-phosphoesterase superfamily } \\
\text { protein }\end{array}$ \\
\hline & Cg9g000380 & -1.08 & undetected & ABC transporter $\mathrm{G}$ family member 37 \\
\hline
\end{tabular}

significantly down-regulated under $\mathrm{Cu}$ excess but upregulated under $\mathrm{Cu}$ deficiency $[20,22,27,42]$. MiR395b and miR395c of $A$. thaliana were found to be upregulated under $\mathrm{Cu}$ and cadmium $(\mathrm{Cd})$ toxicity [43]. In addition, miR166 and miR399 were shown to play important roles in manganese $(\mathrm{Mn}), \mathrm{Cd}$, arsenic (As) or aluminum (Al) toxicity [44]. Although no direct evidence is provided to support the function of miR535 in metal stress, another member sharing same superfamily and high sequence similarity, miR156 has been well documented to function in $\mathrm{Cd}, \mathrm{Al}, \mathrm{Mn}$, and As toxicity by targeting SPL genes [44, 45]. Interestingly, these metal stressrelated miRNAs were predicted to target and regulate a large number of known metal stresses-related genes in the ceRNA network (Fig. 8, Additional file 7: Table S7 and Additional file 8: Table S8). For example, miR398b is predicted to target five DEmRNAs that significantly upregulated by excessive $\mathrm{Cu}$ level, including putative ATPbinding cassette (ABC) transporters (Cg5g003300, Cg6g016060), oligopeptide transporter (OPT, Cg1g001620), multicopper oxidase (MCO, Cg1g015400), and mitogen-activated protein kinase kinase kinase (MAPKKK, Cg8g024350). It is well known that $\mathrm{ABC}$ transporters are one of the largest families of plant proteins that are suggested to be involved in metal ion uptake, transport and heavy metals detoxification [46, 47]. The 

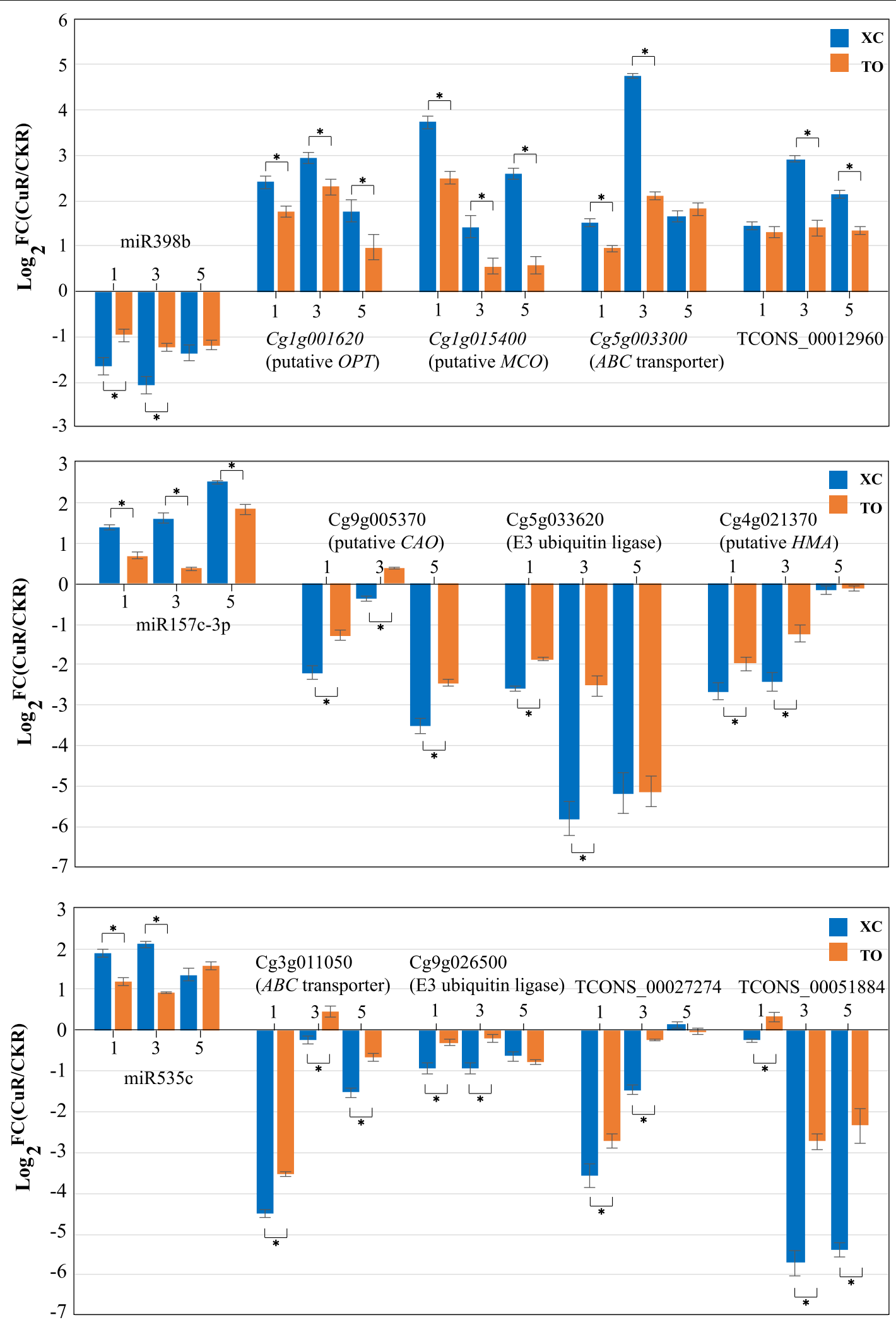

Fig. 9 (See legend on next page.) 
(See figure on previous page.)

Fig. 9 qRT-PCR analysis of the candidate miRNAs, mRNAs and IncRNAs in 'Ziyang Xiangcheng' (XC) and trifoliate orange (TO). Three miRNAs and their predicted targets were selected to determine the expression in roots of $X C$ and TO which were treated with excess and normal Cu for $1 \mathrm{~d}$, $3 \mathrm{~d}$ and $5 \mathrm{~d}$. FC represents the fold changes of the relative expression levels between CuR and CKR. The data are the means \pm SE of three technical replicates. Asterisk $\left(^{*}\right)$ indicates the significant difference at $P<0.05$ by LSD testing

OPT proteins consisting of YSL and PT clades have been demonstrated to participate in metal homeostasis through the translocation of metal-chelates [48]. The MCOs, which belong to the blue $\mathrm{Cu}$ proteins containing one to six $\mathrm{Cu}$ atoms per molecule, are suggested to function in redox reactions [49]. As an important signal transduction pathway, the MAPK signaling cascade plays key roles in response to different extracellular stimuli, and it has been previously found to be activated by excess of $\mathrm{Cu}$ and $\mathrm{Cd}$ $[50,51]$. It is worth mentioning that except the miR398targeted genes, other DEmRNAs, including putative SPLs, YSLs, HMAs, ABC transporters, LACs and ZIPs, were targeted by up-regulated miRNAs. These target mRNAs have also been shown to play essential roles in homeostasis of $\mathrm{Cu}$ or other metals. Therefore, we speculate that the better tolerance of $\mathrm{Cu}$ toxicity in $\mathrm{XC}$ is ascribed, at least in part, to miRNA-mediated regulation of the $\mathrm{Cu}$-related genes, which allows it to maintain $\mathrm{Cu}$ homeostasis through alteration of signal transduction, $\mathrm{Cu}$ uptake, transport and detoxification, and antioxidant capacity. This speculation is corroborated by the differential expression levels of the miRNAs and targeted mRNAs in two genotypes with contrasting tolerance to $\mathrm{Cu}$ toxicity (Fig. 9).

Accumulating evidence indicates that the lncRNAs and circRNAs, two classes of new ncRNAs, act as ceRNAs (or named 'miRNA sponges', 'target mimics') to function in a variety of biological processes. In colorectal cancer, the lncRNA H19 was reported to derepress the endogenous genes, Vimentin, ZEB1, and ZEB2, by targeting miR138 and miR200a [52]. An autophagy promoting factor (APF) lncRNA was shown to interact with miR188-3p, and thus affected ATG7 expression and autophagic cell death in heart [53]. In plants, overexpression of lncRNA IPS1 in A. thaliana led to increased accumulation of $\mathrm{PHO} 2$, which is targeted by miR399 and functions in phosphate uptake [54]. In addition, circRNAs have been revealed to function as miRNA sponges. For example, a circHIPK3 could sponge to nine miRNAs with 18 potential binding sites and significantly affected human cell growth [55]. In this study, we

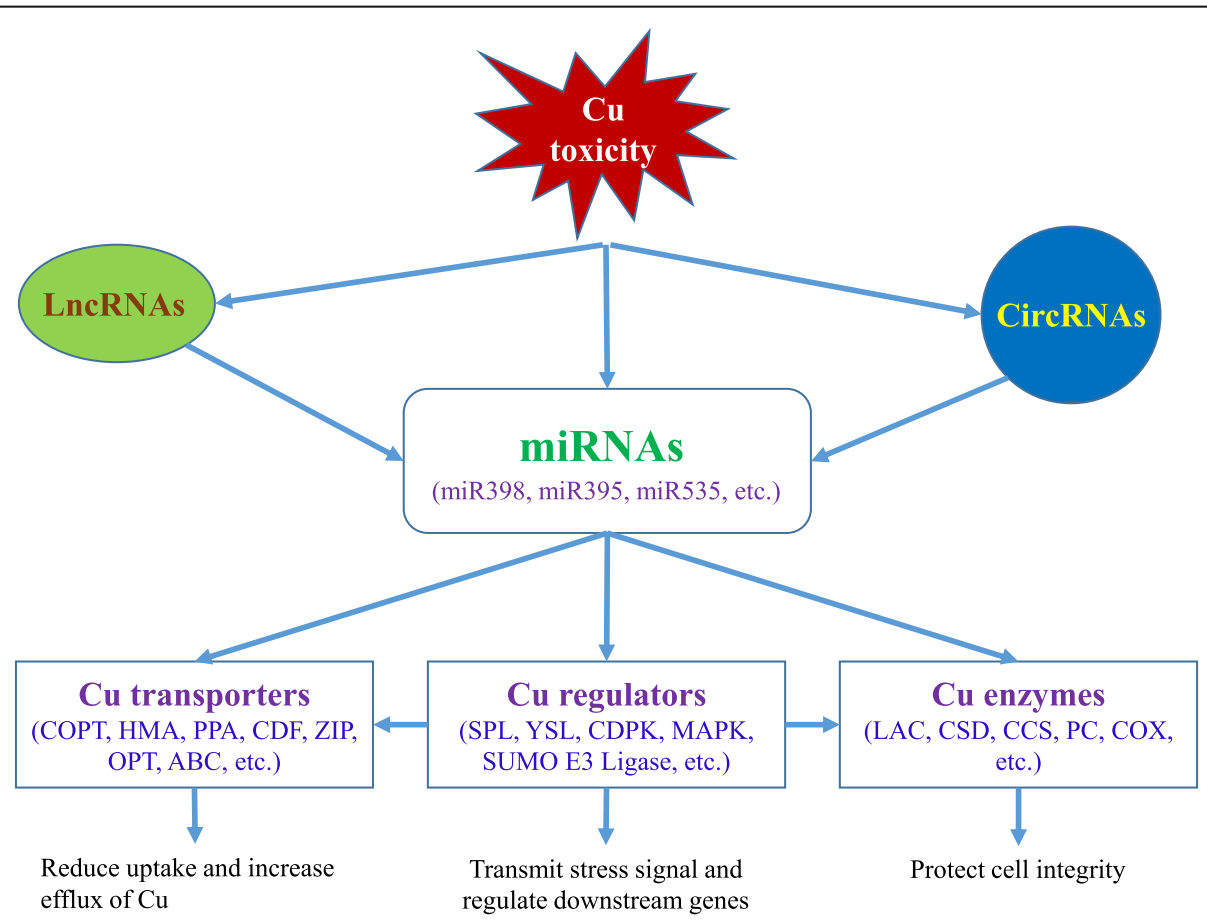

Fig. 10 The proposed model in response to Cu toxicity in citrus. Under Cu toxicity, miRNAs act as the key regulators that directly target important Cu transporters, Cu proteins and Cu regulators (TFs and kinases). In this process, some IncRNAs and circRNAs can act as ceRNA to competitively bind the MRE of miRNAs, which may indirectly affect the expression of mRNAs 
identified nine DElncRNAs in the ceRNA network, suggesting that these citrus lncRNAs may also function as miRNA sponges to participate in tolerance to $\mathrm{Cu}$ toxicity. However, it should be pointed out that most of DElncRNAs were not included in the ceRNA network. It is thus assumed that these IncRNAs involve in $\mathrm{Cu}$ toxicity via other mechanisms, such as transcribing as miRNA precursors, induction of DNA methylation, modulation of chromatin modification, or serving as transcription enhancers [56]. It is surpring and unexpected that all of the DEcircRNAs were not included in the ceRNA network. One of the possible reasons is that the criteria used to predict the ceRNA pairs and interactions between DERNAs is too stringent.

Based on the ceRNA theory and the ceRNA network constructed in this study, we propose a mode of action for the differentially expressed mRNAs, miRNAs, lncRNAs and circRNAs in response to the $\mathrm{Cu}$ toxicity (Fig. 10). Under $\mathrm{Cu}$ toxicity, miRNAs act as the key regulators to modulate up-regulation or down-regulation of important $\mathrm{Cu}$ transporters, $\mathrm{Cu}$ proteins and $\mathrm{Cu}$ regulators (TFs and kinases). As a consequence, uptake, efflux and distribution of $\mathrm{Cu}$ will be activated or suppressed, and cell integrity was protected, leading to enhanced tolerance to $\mathrm{Cu}$ toxicity. In this process, some lncRNAs can act as ceRNA to competitively bind the MRE of miRNAs, which may indirectly affect the expression of mRNA. Moreover, circRNAs possibly act as another type of ceRNA to sequester $\mathrm{Cu}$-responsive miRNAs and suppress their function.

\section{Conclusions}

Tolerance evaluation showed that XC was most tolerant to $\mathrm{Cu}$ toxicity. Whole-transcriptome RNAseq helped us to identify 5734 (2162 up-regulated, 3572 down-regulated) and 222 (132 up-regulated, 90 down-regulated) DEmRNAs in Cu-treated root and leaf, respectively, in which 1243 were considered to be key candidates in response to excessive $\mathrm{Cu}$. We also identified 243 known and 1033 novel lncRNAs, of which 164 (103 up-regulated, 61 downregulated) and 5 (1 up-regulated, 4 down-regulated) were significantly differentially expressed in the $\mathrm{Cu}$-treated root and leaf, respectively. From 2404 identified circRNAs, only 45 (28 up-regulated, 17 down-regulated) and 17 (11 upregulated, 6 down-regulated) DEcircRNAs were identified in root and leaf, respectively, exposed to excessive $\mathrm{Cu}$. In addition, 149 known miRNAs and 336 novel miRNAs were predicted, and 147 and 130 of them were responsive to $\mathrm{Cu}$ toxicity in the root and leaf, respectively. GO enrichment analysis showed that most of the DEmRNAs and targets of DElncRNAs and DEmiRNAs were implicated in oxidation-reduction, phosphorylation, membrane, and ion binding. A ceRNA network consisting of differentially expressed mRNAs, miRNAs, and IncRNAs was constructed, which further revealed the critical roles of these DERNAs in tolerance to $\mathrm{Cu}$ toxicity.

\section{Methods \\ Plant materials and treatments}

Seeds of four commonly used citrus rootstocks, trifoliate orange (Poncirus trifoliata L. Raf.), 'Ziyang Xiangcheng' (Citrus junos Sieb. ex Tanaka), red tangerine (C. reticulata Blanco), and 'Shatian' pummelo (C. grandis) were collected from Citrus Research Institute of Southwest University (Chongqing, China). To evaluate the tolerance to $\mathrm{Cu}$ toxicity, seeds of the rootstocks were germinated in plastic containers filled with quartz sand at a temperature of $28{ }^{\circ} \mathrm{C}$ and a relative humidity of $80 \%$. Uniform seedlings were transplanted into fresh quartz sand washed with distilled water at $25^{\circ} \mathrm{C}$ under a $16-\mathrm{h}$ photoperiod $\left(50 \mu \mathrm{mol} \cdot \mathrm{m}^{-2} \cdot \mathrm{s}^{-1}\right)$ for sand culture. During sand culture, $1 / 2$-strength Hoagland's solution composed of $4 \mathrm{mM} \mathrm{Ca}\left(\mathrm{NO}_{3}\right)_{2}, 1.5 \mathrm{mM} \mathrm{KNO}_{3}, 0.5 \mathrm{mM} \mathrm{NH}_{4} \mathrm{H}_{2} \mathrm{PO}_{4}$, $1 \mathrm{mM} \mathrm{MgSO}_{4}, 50 \mu \mathrm{M}$ Fe-EDTA, $15 \mu \mathrm{M} \mathrm{H}_{3} \mathrm{BO}_{3}, 10 \mu \mathrm{M}$ $\mathrm{MnSO}_{4}, \quad 5 \mu \mathrm{M} \quad \mathrm{ZnSO}_{4}, \quad 1.5 \mu \mathrm{M} \quad \mathrm{CuSO}_{4}$, and $1 \mu \mathrm{M}$ $\mathrm{H}_{2} \mathrm{MoO}_{4}$, was irrigated every $4 \mathrm{~d}$. After $30 \mathrm{~d}$ of growth, half of the seedlings were irrigated with the above mentioned solution (used as control, CK), while the rest seedlings were irrigated with $1 / 2$-strength Hoagland's solution containing $187.5 \mu \mathrm{M} \mathrm{CuSO}_{4}(125 \times)$ for $40 \mathrm{~d}$, which was considered as $\mathrm{Cu}$ treatment. Three biological replicates were performed for each treatment.

For RNAseq and quantitative real time PCR (qRT-PCR) validation, seeds of 'Ziyang Xiangcheng' and trifoliate orange were first sterilized with $2 \%$ sodium hypochlorite for $15 \mathrm{~min}$. After removal of seed coats, the seeds were germinated at a temperature of $28^{\circ} \mathrm{C}$ and a relative humidity of $80 \%$ for one week. Uniform seedlings were transferred to the above-mentioned normal $1 / 2$-strength Hoagland's solution for hydroculture at $25^{\circ} \mathrm{C}$ under a 16$\mathrm{h}$ photoperiod $\left(50 \mu \mathrm{mol} \cdot \mathrm{m}^{-2} \cdot \mathrm{s}^{-1}\right)$, and the solution was renewed every $10 \mathrm{~d}$. After $30 \mathrm{~d}$ of growth, half of the seedlings were renewed with $1 / 2$-strength Hoagland's solution containing $75 \mu \mathrm{M} \mathrm{CuSO}_{4}(50 \times)$ for excess $\mathrm{Cu}$ treatment, while the others were renewed with normal solution (CK). After $1 \mathrm{~d}, 3 \mathrm{~d}$ and $5 \mathrm{~d}$ of treatment, the roots and leaves of the seedlings were sampled, frozen in liquid nitrogen, and stored at $-80^{\circ} \mathrm{C}$, respectively. Three biological replicates were performed for each treatment.

\section{Measurement of plant height and contents of chlorophyll and malonaldehyde}

Plant height $(\mathrm{PH})$ of the aerial part was measured with a ruler at 0 (PH1) and $40 \mathrm{~d}$ of $\mathrm{Cu}$ treatment $(\mathrm{PH} 2)$. Relative increase rate of the plant height was calculated as $(\mathrm{PH} 2-\mathrm{PH} 1) / \mathrm{PH} 1 \times 100 \%$. Contents of chlorophyll and malonaldehyde (MDA) were determined as described by $\mathrm{Fu}$ et al. [57]. 
RNA extraction, library preparation, and RNA sequencing Total RNA was extracted from the roots and leaves of CK (abbreviated as CKR and CKL) and Cu-treated samples (abbreviated as $\mathrm{CuR}$ and $\mathrm{CuL}$ ) using TRIzol Reagent (Invitrogen, Carlsbad, CA, USA) according to the manufacturer's protocol. RNA quality and integrity were estimated with an Agilent 2100 Bioanalyzer (Agilent Technologies, Santa Clara, CA, USA) and NanoDrop 2000 spectrophotometer (Thermo Scientific, Wilmington, DE, USA). Only high-quality RNA samples $(1.8<$ OD $260 / 280<2.2, \quad$ OD $260 / 230 \geq 2.0, \quad$ RIN $\geq 7.0, \quad 28 \mathrm{~S} /$ $18 \mathrm{~S} \geq 1.0$ ) were used to construct the sequencing library.

For mRNA, LncRNA, and circRNA sequencing, $5 \mu \mathrm{g}$ of total RNA was used to prepare ribosomal RNA (rRNA) removed strand-specific library using a TruSeq Stranded Total RNA Library Prep with the Ribo-Zero Plant Kit (Illumina, San Diego, CA, USA) according to the manufacturer's instructions. There were three biological replicates per treatment, and a total of 12 libraries were prepared. For small RNA sequencing, 4 small RNA libraries were constructed with $3 \mu \mathrm{g}$ of total RNA from CKL, CKR, CuL and CuR samples and the Truseq Small RNA sample prep Kit (Illumina, San Diego, CA, USA). After libraries were quantified by a TBS-380 Fluorometer (Turner Biosystem, Sunnyvale, CA, USA), deep RNA sequencing was performed using an Illumina HiSeq X Ten platform at Shanghai Majorbio Bio-Pharm Biotechnology Co. Ltd. (Shanghai, China).

\section{Read mapping and transcriptome assembly}

The paired-end raw reads were trimmed and quality controlled by SeqPrep (version 1.1, https://github.com/ jstjohn/SeqPrep) and Sickle (version 1.33, https://github. com/najoshi/sickle) with default parameters. Then, clean reads were separately aligned to the Pummelo (Citrus grandis) genome [39] in orientation mode using Tophat2 software [58] (version 2.0.13, http://tophat.cbcb.umd. $\mathrm{edu} /$ ). The mapped reads of each sample were assembled by Cufflink (version 2.2.1, http://cufflinks.cbcb.umd.edu/ ) in a reference-based approach.

\section{Differentially expressed mRNA and gene ontology (GO) enrichment analysis}

To identify differentially expressed mRNAs (DEmRNAs) between $\mathrm{CK}$ and $\mathrm{Cu}$-treated samples, the expression level of each transcript was calculated according to the fragments per kilobase of exon per million mapped reads (FRKM) method. RSEM (version 1.2.31, http://deweylab.biostat.wisc.edu/rsem/) was used to quantify gene abundance [59]. R statistical package software EdgeR (version 3.14.0) [60] was utilized for differential expression analysis with an absolute value of $\log _{2} \mathrm{FC}>1$ and $\mathrm{FDR}<0.05$. GO annotation and functional enrichment analysis of
DEmRNAs were carried out using the Omicshare oneline platform (http://www.omicshare.com/tools/).

\section{Identification of IncRNAs and prediction of their target genes}

For identification of novel lncRNAs, the transcripts that overlapped with known protein-coding genes on the same strand, transcripts with a fragment count < 3 , transcripts shorter than $200 \mathrm{nt}$, and an open reading frame (ORF) longer than $300 \mathrm{nt}$ were first discarded [61, 62]. Then, we used the Coding Potential Calculator (CPC, version 0.9), Coding-Non-Coding index (CNCI, version 2.0), Coding Potential Accessment Tool (CPAT, version 1.2.4), and Pfam Scan (version 1.6) to filter transcripts with coding potential. The overlapped outputs from CPC, CNCI, CPAT, and Pfam Scan were considered reliably expressed novel lncRNAs. The transcripts were also used to blast the citrus lncRNA sequences that were collected in the GREENC database (http://greenc.sciencedesigners. com/wiki/Main_Page), and the hits with e_value $<1 \mathrm{E}$ 5 and matched bases ratio $>90 \%$ were identified as know lncRNAs. All identified lncRNAs were classified into intergenic, intronic, and antisense lncRNAs using the cuffcompare program in the Cufflinks suite. The expression level of each lncRNA was calculated according to the FRKM method. Differentially expressed lncRNAs (DELncRNAs) were extracted with an absolute value of $\log _{2} \mathrm{FC}>1$ and $\mathrm{FDR}<0.05$ by EdgeR. The potential cis- and trans-target mRNAs of DELncRNAs were predicted according to the position on the chromosome. The cis-targets were searched within a 10-kb window upstream or downstream of the lncRNA [63]. The trans-targets were predicted as described by $\mathrm{Ou}$ et al. [64].

\section{Identification and analysis of circRNAs}

CircRNA Identifier (CIRI, version 1.2) and CIRCexplorer (version 1.1.7) tools were used to identify circRNAs in this study. CIRI scans Sequence Alignment/ Map (SAM) files and detects junction reads with paired chiastic clipping (PCC) signals and paired-end mapping (PEM) and GT-AG splicing signals as described by Gao et al. [65]. CIRCexplorer obtains junction reads via a two-step TopHat and TopHat-Fusion mapping strategy as described by Zhang et al. [66] The overlapped outputs from CIRI and CIRCexplorer were used for further analysis. The expression level of each circRNA was calculated according to the Spliced Reads per Billion Mapping (SRPBM) method. Differentially expressed circRNAs (DEcircRNAs) were extracted with an absolute value of $\log _{2} \mathrm{FC}>1$ and FDR $<0.05$ by DEGseq (version 1.30.0). 


\section{Identification and analysis of miRNAs}

The raw data were first quality controlled with Fastxtoolkit software (version. 0.0.13, http://hannonlab.cshl. edu/fastx_toolkit/) to obtain clean small RNA reads by filtering low-quality bases (sanger base quality <20), sequencing adapters, reads shorter than $18 \mathrm{nt}$, and reads longer than $32 \mathrm{nt}$. The assembled unique sequences with clean reads were then BLAST searched against the Rfam database (version 12.1, http://rfam.sanger.ac.uk/) to remove non-miRNA sequences (rRNA, tRNA, snoRNA, etc.). The remaining reads were used to predict known miRNAs through a BLAST search of the miRbase, version 21.0 (http://www.mirbase.org/), and novel miRNAs through analysis of the hairpin structure of the miRNA precursor with Mireap (version 0.2, http://sourceforge. net/projects/mireap) software. The expression level of each miRNA was calculated according to the transcripts per million reads (TPM) method. Differentially expressed miRNAs (DEmiRNAs) were extracted with an absolute value of $\log _{2} \mathrm{FC}>1$ and $\mathrm{FDR}<0.005$ by DEGseq. Target prediction of DEmiRNAs was performed with psRobot local version 1.01 [67].

\section{Construction and analysis of ceRNAs regulatory network}

To reveal the roles and interactions of lncRNAs, circRNAs, miRNAs, and mRNAs, we constructed a lncRNAcircRNA-miRNA-mRNA regulatory network based on the ceRNA hypothesis. psRobot [67] was used to predict the pairs of miRNA-IncRNA, miRNA-mRNA, and miRNAcircRNA. The pairwise correlations of miRNA-lncRNA, miRNA-mRNA and miRNA-circRNA were evaluated using the Spearman correlation coefficient (SCC) and the matched pairs' expression profile data as described by $\mathrm{He}$ et al. [40]. The interaction network was built with RNA pairs of SCC $<-0.5$ and visually displayed using Cytoscape software (version 2.8) [68].

\section{qRT-PCR validation of DEmRNAs, DElncRNAs, and DEmiRNAs}

qRT-PCR was performed to validate the expression levels of DEmRNAs, DElncRNAs, and DEmiRNAs. Total RNA and small RNA were extracted from samples of $\mathrm{CuR}$ and CKR using an RNAprep pure Plant Kit (TIANGEN, Cat\#DP432, China) and miRcute miRNA isolation kit (TIANGEN, Cat\#DP501, China), respectively. Firststrand cDNA was synthesized from $1 \mu \mathrm{g}$ of total RNA with the HiScript $^{\circ}$ II Q RT SuperMix (Vazyme, Cat\#R223, China) for qPCR of mRNA and with the lnRcute lncRNA First-Strand cDNA Synthesis Kit (TIANGEN, Cat\#KR202, China) for qPCR of lncRNA. In addition, $1 \mu \mathrm{g}$ of small RNA was used for cDNA synthesis using a miRNA 1st Strand cDNA Synthesis Kit (Vazyme, Cat\#MR101, China) with the stem-loop primer designed by the stem-loop sequence (GTCGTATCCA
GGGTCCGAGGTATTCGCACTGGATACGAC) except for the internal reference U6. qPCR was performed on the Bio-Rad CFX Connect RealTime system using ChamQ $^{\text {ts }}$ Universal SYBR ${ }^{\odot}$ qPCR Master Mix (Vazyme, Cat\#Q711), lnRcute IncRNA qPCR Detection Kit (TIANGEN, Cat\#FP402) and miRNA Universal SYBR ${ }^{\circ}$ qPCR Master Mix (Vazyme, Cat\#MQ101) following the manufacturer's instructions. The $2^{-\Delta \Delta C T}$ method was used to normalize and determine the RNA level relative to an internal reference gene, actin (Cs1g05000.1) or U6. All primers are included in Additional file 9: Table S9.

\section{Supplementary information}

Supplementary information accompanies this paper at https://doi.org/10. 1186/s12870-019-2087-1.

Additional file 1: Table S1. List of 5734 and 222 DEmRNAs in the root and leaf, respectively.

Additional file 2: Table S2. List of 164 and 5 DElncRNAs in the root and leaf, respectively.

Additional file 3: Table S3. Cis and trans targets of DElncRNAs.

Additional file 4: Table S4. List of 45 and 17 DEcircRNAs in the root and leaf, respectively.

Additional file 5: Table S5. List of 147 and 130 DEmiRNAs in the root and leaf, respectively.

Additional file 6: Table S6. DEmiRNAs targeted DEmRNAs in the root and leaf, respectively.

Additional file 7: Table S7. Searched known Cu-related DEmRNAs by using the keywords reported in previous studies to query their functional descriptions.

Additional file 8: Table S8. List of 1273 key DEmRNAs in response to Cu toxicity in citrus.

Additional file 9: Table S9. List of primers used in qRT-PCR.

Additional file 10: Figure S1. GO annotation of DEmRNAs in the leaf (a) and root (b), and GO enrichment of DEmRNAs in the leaf (c).

Additional file 11: Figure S2. GO annotation of targets of DElncRNAs in the root.

Additional file 12: Figure S3. GO annotation of targets of known (a) and novel (b) DEmiRNAs in the root.

\footnotetext{
Abbreviations

ABC transporter: ATP-binding cassette transporter; BP: Biological process; CC: Cellular component; ceRNAs: competing endogenous RNAs; circRNAs: circular RNAs; CIRI: circRNA identifier; CKL: Cu-untreated leaf; CKR: Cu-untreated root; CNCl: Coding-non-coding index; COPTs: CTR-like Cu transporters; COX: Cytochrome c oxidase; CPAT: Coding potential accessment tool; CPC: Coding potential calculator; CSD: Copper/zinc superoxide dismutases; Cu: Copper; CuL: Cu-treated leaf; CuR: Cu-treated root; DAO: Diamine oxidases; DE: Differentially expressed; FC: Fold change; FRKM: The fragments per kilobase of exon per million mapped reads; GO: Gene ontology; HMAs: P-type heavy metal ATPases; LAC: Laccase; IncRNAs: Long non-coding RNAs; MAPKKK: Mitogen-activated protein kinase kinase kinase; MCO: Multicopper oxidase; MDA: Malonaldehyde; MF: Molecular function; miRNAs: microRNAs; MREs: miRNA response elements; OPT: Oligopeptide transporter; ORF: Open reading frame; PC: Plastocyanin; PCC: Paired chiastic clipping; PEM: Paired-end mapping; PH: Plant height; qRT-PCR: Quantitative real time PCR; RNAseq: RNA sequencing; SAM: Sequence alignment/map; SCC: Spearman correlation coefficient; SPL7: Squamosa promoter binding-like 7; SRPBM: The spliced reads per billion mapping; TFs: Transcription factors; TO: Trifoliate orange TPM: Transcripts per million reads; XC: 'Ziyang Xiangcheng'; YSL: Yellow stripe-like protein
} 


\section{Acknowledgements}

We are grateful to prof. Jihong Liu from Huazhong Agricultural University for his revision of the manuscript.

\section{Authors' contributions}

XZF and LZP conceived and designed the study. XZF, XYZ, and YZH analyzed the data. JYQ and XZ performed qRT-PCR. MY and LC prepared experimental materials. CPC and LLL measured physiological data. XZF wrote the paper. All authors read and approved the final manuscript.

\section{Funding}

This work was financially supported by the National Key Research and Development Program of China (2018YFD1000300, 2017YFD0202000), the National Natural Science Foundation of China (31772280), and the National Citrus Engineering Research Center (NCERC2019001). The funders provided the financial support to the RNAseq, but didn't play role in in the design of the study, collection, analysis, interpretation of data, and in writing the manuscript.

\section{Availability of data and materials}

All supporting data can be found within the manuscript and its additional supporting files.

\section{Ethics approval and consent to participate}

Not applicable.

\section{Consent for publication}

Not applicable.

\section{Competing interests}

The authors declare that they have no competing interests.

\section{Author details}

'Citrus Research Institute, Southwest University, Chongqing 400712, China. ${ }^{2}$ Citrus Research Institute, Chinese Academy of Agricultural Sciences, Chongqing 400712, China.

Received: 8 August 2019 Accepted: 20 October 2019

Published online: 21 November 2019

\section{References}

1. Broadley M, Brown P, Cakmak I, Rengel Z, Zhao F. Function of nutrients: Micronutrients. p. 191-248. In: P. Marschner (eds.), Mineral Nutrition of Higher Plants, Elsevier, 2012.

2. Burkhead JL, Reynolds KAG, Abdel-Ghany SE, Cohu CM, Pilon M. Copper homeostasis. New Phytol. 2009;182:799-816.

3. Yruela Guerrero I. Copper in plants: acquisition, transport and interactions. Funct Plant Biol. 2009:36:409-30.

4. Yan J, Chia JC, Sheng H, Jung HI, Zavodna TO, Zhang L, Huang R, Jiao C, Craft EJ, Fei Z, et al. Arabidopsis pollen fertility requires the transcription factors CITF1 and SPL7 that regulate copper delivery to anthers and jasmonic acid synthesis. Plant Cell. 2017;29:3012-29.

5. Cambrolle J, Garcia JL, Figueroa ME, Cantos M. Evaluating wild grapevine tolerance to copper toxicity. Chemosphere. 2015;120:171-8.

6. Leng X, Jia H, Sun X, Shangguan L, Mu Q, Wang B, Fang J. Comparative transcriptome analysis of grapevine in response to copper stress. Sci Rep. 2015:5:17749.

7. Huang XY, Deng F, Yamaji N, Pinson SR, Fujii-Kashino M, Danku J, Douglas A, Guerinot ML, Salt DE, Ma JF. A heavy metal P-type ATPase OsHMA4 prevents copper accumulation in rice grain. Nat Commun. 2016;7:12138.

8. Zhao FJ, Ma Y, Zhu YG, Tang Z, McGrath SP. Soil contamination in China: current status and mitigation strategies. Environ Sci Technol. 2015;49:750-9.

9. Clemens S. Molecular mechanisms of plant metal tolerance and homeostasis. Planta. 2001;212:475-86.

10. Clemens S, Palmgren MG, Kramer U. A long way ahead: understanding and engineering plant metal accumulation. Trends Plant Sci. 2002;7:309-15.

11. Aguirre G, Pilon M. Copper delivery to chloroplast proteins and its regulation. Front Plant Sci. 2016;6:1250.

12. Sancenon V, Puig S, Mateu-Andres I, Dorcey E, Thiele DJ, Penarrubia L. The Arabidopsis copper transporter COPT1 functions in root elongation and pollen development. J Biol Chem. 2004;279:15348-55.
13. Jung HI, Gayomba SR, Rutzke MA, Craft E, Kochian LV, Vatamaniuk OK COPT6 is a plasma membrane transporter that functions in copper homeostasis in Arabidopsis and is a novel target of SQUAMOSA promoterbinding protein-like 7. J Biol Chem. 2012;287:33252-67.

14. Andres-Colas N, Sancenon V, Rodriguez-Navarro S, Mayo S, Thiele DJ, Ecker JR, Puig S, Penarrubia L. The Arabidopsis heavy metal P-type ATPase HMA5 interacts with metallochaperones and functions in copper detoxification of roots. Plant J. 2006:45:225-36.

15. Shikanai T, Muller-Moule P, Munekage Y, Niyogi KK, Pilon M. PAA1, a P-type ATPase of Arabidopsis, functions in copper transport in chloroplasts. Plant Cell. 2003:15:1333-46.

16. Abdel-Ghany SE, Muller-Moule P, Niyogi KK, Pilon M, Shikanai T. Two P-type ATPases are required for copper delivery in Arabidopsis thaliana chloroplasts. Plant Cell. 2005;17:1233-51.

17. Zheng L, Yamaji N, Yokosho K, Ma JF. YSL16 is a phloem-localized transporter of the copper-nicotianamine complex that is responsible for copper distribution in rice. Plant Cell. 2012;24:3767-82.

18. Yamasaki H, Hayashi M, Fukazawa M, Kobayashi Y, Shikanai T. SQUAMOSA promoter binding protein-like 7 is a central regulator for copper homeostasis in Arabidopsis. Plant Cell. 2009;21:347-61.

19. Araki R, Mermod M, Yamasaki H, Kamiya T, Fujiwara T, Shikanai T. SPL7 locally regulates copper-homeostasis-related genes in Arabidopsis. J Plant Physiol. 2018;224:137-43.

20. Yamasaki H, Abdel-Ghany SE, Cohu CM, Kobayashi Y, Shikanai T, Pilon M. Regulation of copper homeostasis by micro-RNA in Arabidopsis. J Biol Chem. 2007:282:16369-78.

21. Bernal M, Casero D, Singh V, Wilson GT, Grande A, Yang H, Dodani SC, Pellegrini M, Huijser $\mathrm{P}$, Connolly EL, et al. Transcriptome sequencing identifies SPL7-regulated copper acquisition genes FRO4/FRO5 and the copper dependence of iron homeostasis in Arabidopsis. Plant Cell. 2012;24:738-61

22. Pilon M. The copper microRNAs. New Phytol. 2017;213:1030-5.

23. Wang J, Meng X, Dobrovolskaya OB, Orlov YL, Chen M. Non-coding RNAs and their roles in stress response in plants. Genom Proteom Bioinf. 2017;15:301-12.

24. Chien PS, Chiang CB, Wang Z, Chiou TJ. MicroRNA-mediated signaling and regulation of nutrient transport and utilization. Curr Opin Plant Biol. 2017:39:73-9.

25. Abdel-Ghany SE, Pilon M. MicroRNA-mediated systemic down-regulation of copper protein expression in response to low copper availability in Arabidopsis. J Biol Chem. 2008;283:15932-45.

26. Lu S, Li Q, Wei H, Chang M, Tunlaya-Anukit S, Kim H, Liu J, Song J, Sun $\mathrm{YH}$, Yuan L, et al. Ptr-miR397a is a negative regulator of laccase genes affecting lignin content in Populus trichocarpa. P Natl Acad Sci USA. 2013:110:10848-53.

27. Sunkar R, Kapoor A, Zhu JK. Posttranscriptional induction of two cu/Zn superoxide dismutase genes in Arabidopsis is mediated by downregulation of miR398 and important for oxidative stress tolerance. Plant Cell. 2006;18: 2051-65.

28. Chekanova JA. Long non-coding RNAs and their functions in plants. Curr Opin Plant Biol. 2015:27:207-16.

29. Ma K, Shi W, Xu M, Liu J, Zhang F. Genome-wide identification and characterization of long non-coding RNA in wheat roots in response to $\mathrm{Ca}^{2+}$ channel blocker. Front Plant Sci. 2018;9:244.

30. Li QF, Zhang YC, Chen YQ, Yu Y. Circular RNAs roll into the regulatory network of plants. Biochem Bioph Res. 2017;488:382-6.

31. Salmena L, Poliseno L, Tay $Y$, Kats L, Pandolfi PP. A ceRNA hypothesis: the Rosetta stone of a hidden RNA language? Cell. 2011;146:353-8.

32. Xu XW, Zhou XH, Wang RR, Peng WL, An Y, Chen LL. Functional analysis of long intergenic non-coding RNAs in phosphate-starved rice using competing endogenous RNA network. Sci Rep. 2016;6:20715.

33. Zhu M, Zhang M, Xing L, Li W, Jiang $H$, Wang $L$, Xu M. Transcriptomic analysis of long non-coding RNAs and coding genes uncovers a complex regulatory network that is involved in maize seed development. Genes. 2017:8:274.

34. Ren GJ, Fan XC, Liu TL, Wang SS, Zhao GH. Genome-wide analysis of differentially expressed profiles of mRNAs, IncRNAs and circRNAs during Cryptosporidium baileyi infection. BMC Genomics. 2018;19:356.

35. Meng X, Zhang P, Chen Q, Wang J, Chen M. Identification and characterization of ncRNA-associated ceRNA networks in Arabidopsis leaf development. BMC Genomics. 2018;19:607. 
36. Yuan Y, Li J, Xiang W, Liu Y, Shu J, Gou M, Qing M. Analyzing the interactions of mRNAs, miRNAs, IncRNAs and circRNAs to predict competing endogenous RNA networks in glioblastoma. J Neuro-Oncol. 2018;137:493-502.

37. Yang Z, Yang C, Wang Z, Yang Z, Chen D, Wu Y. LncRNA expression profile and ceRNA analysis in tomato during flowering. PLoS One. 2019;14: e0210650.

38. Wang Y, Wang Q, Gao L, Zhu B, Luo Y, Deng Z, Zuo J. Integrative analysis of circRNAs acting as ceRNAs involved in ethylene pathway in tomato. Physiol Plantarum. 2017:161:311-21.

39. Wang $X, X u$ Y, Zhang S, Cao L, Huang Y, Cheng J, Wu G, Tian S, Chen C, Liu $Y$, et al. Genomic analyses of primitive, wild and cultivated citrus provide insights into asexual reproduction. Nat Genet. 2017:49:765-72.

40. He X, Guo S, Wang Y, Wang L, Shu S, Sun J, et al. Physiol Plantarum. 2019. https://doi.org/10.1111/ppl.12997.

41. Su XQ, Xing JD, Wang ZZ, Chen L, Cui M, Jiang BH. microRNAs and ceRNAs: RNA networks in pathogenesis of cancer. Chinese J Cancer Res. 2013;25:235-9.

42. Leng $X$, Wang $P$, Zhao $P$, Wang M, Cui L, Shangguan L, Wang C. Conservation of microRNA-mediated regulatory networks in response to copper stress in grapevine. Plant Growth Regul. 2017:82:293-304.

43. Gielen H, Remans T, Vangronsveld J, Cuypers A. Toxicity responses of $\mathrm{Cu}$ and cd: the involvement of miRNAs and the transcription factor SPL7. BMC Plant Biol. 2016;16:145.

44. Gupta OP, Sharma P, Gupta RK, Sharma I. MicroRNA mediated regulation of metal toxicity in plants: present status and future perspectives. Plant Mol Biol. 2014;84:1-18

45. Ding Y, Chen Z, Zhu C. Microarray-based analysis of cadmium-responsive microRNAs in rice (Oryza sativa). J Exp Bot. 2011;62:3563-73.

46. Wojas S, Hennig J, Plaza S, Geisler M, Siemianowski O, Sklodowska A, Ruszczynska A, Bulska E, Antosiewicz DM. Ectopic expression of Arabidopsis ABC transporter MRP7 modifies cadmium root-to-shoot transport and accumulation. Environ Pollut. 2009:157:2781-9.

47. Kim DY, Bovet L, Maeshima M, Martinoia E, Lee Y. The ABC transporter AtPDR8 is a cadmium extrusion pump conferring heavy metal resistance. Plant J. 2007:50:207-18

48. Lubkowitz M. The oligopeptide transporters: a small gene family with a diverse group of substrates and functions? Mol Plant. 2011;4:407-15.

49. Hoegger PJ, Kilaru S, James TY, Thacker JR, Kues U. Phylogenetic comparison and classification of laccase and related multicopper oxidase protein sequences. FEBS J. 2006:273:2308-26.

50. Jonak C, Nakagami H, Hirt H. Heavy metal stress. Activation of distinct mitogen-activated protein kinase pathways by copper and cadmium. Plant Physiol. 2004;136:3276-83.

51. Yeh CM, Hsiao LJ, Huang HJ. Cadmium activates a mitogen-activated protein kinase gene and MBP kinases in rice. Plant Cell Physiol. 2004;45: 1306-12.

52. Liang WC, Fu WM, Wong CW, Wang Y, Wang WM, Hu GX, Zhang L, Xiao LJ, Wan DCC, Zhang JF, et al. The IncRNA H19 promotes epithelial to mesenchymal transition by functioning as miRNA sponges in colorectal cancer. Oncotarget. 2015;6:22513-25.

53. Wang K, Liu CY, Zhou LY, Wang JX, Wang M, Zhao B, Zhao WK, XU SJ, Fan LH, Zhang XJ, et al. APF IncRNA regulates autophagy and myocardial infarction by targeting miR-188-3p. Nat Commun. 2015;6:6779.

54. Franco-Zorrilla JM, Valli A, Todesco M, Mateos I, Puga MI, Rubio-Somoza I, Leyva A, Weigel D, Garcia JA, Paz-Ares J. Target mimicry provides a new mechanism for regulation of microRNA activity. Nat Genet. 2007;39:1033-7.

55. Zheng QP, Bao CY, Guo WJ, Li SY, Chen J, Chen B, Luo YT, Lyu DB, Li $Y$, Shi GH, et al. Circular RNA profiling reveals an abundant circHIPK3 that regulates cell growth by sponging multiple miRNAs. Nat Commun. 2016;7:11215

56. Hou J, Lu D, Mason AS, Li B, Xiao M, An S, Fu D. Non-coding RNAs and transposable elements in plant genomes: emergence, regulatory mechanisms and roles in plant development and stress responses. Planta. 2019:50:23-40.

57. Fu XZ, Khan EU, Hu SS, Fan QJ, Liu JH. Overexpression of the betaine aldehyde dehydrogenase gene from Atriplex hortensis enhances salt tolerance in the transgenic trifoliate orange (Poncirus trifoliata L. Raf.). Environ Exp Bot. 2011:74:106-13.

58. Kim D, Pertea G, Trapnell C, Pimentel H, Kelley R, Salzberg SL. TopHat2: accurate alignment of transcriptomes in the presence of insertions, deletions and gene fusions. Genome Biol. 2013;14:R36.
59. Li B, Dewey CN. RSEM: accurate transcript quantification from RNA-Seq data with or without a reference genome. BMC Bioinformatics. 2011:12:323.

60. Robinson MD, McCarthy DJ, Smyth GK. EdgeR: a bioconductor package for differential expression analysis of digital gene expression data. Bioinformatics. 2010;26:139-40.

61. Ding ZH, Wu CL, Tie WW, Yan Y, He GY, Hu W. Strand-specific RNA-seq based identification and functional prediction of IncRNAs in response to melatonin and simulated drought stresses in cassava. Plant Physiol Bioch. 2019;140:96-104.

62. Quinn JJ, Chang HY. Unique features of long non-coding RNA biogenesis and function. Nat Rev Genet. 2016:17:47-62.

63. Jia H, Osak M, Bogu GK, Stanton LW, Johnson R, Lipovich L. Genome-wide computational identification and manual annotation of human long noncoding RNA genes. RNA. 2010;16:1478-87.

64. Ou L, Liu Z, Zhang Z, Wei G, Zhang Y, Kang L, Yang B, Yang S, LV J, Liu Y, et al. Noncoding and coding transcriptome analysis reveals the regulation roles of long noncoding RNAs in fruit development of hot pepper (Capsicum annuum L.). Plant Growth Regul. 2017:83:1-16.

65. Gao Y, Wang J, Zhao F. CIRl: an efficient and unbiased algorithm for de novo circular RNA identification. Genome Biol. 2015:16:4.

66. Zhang XO, Wang HB, Zhang Y, Lu X, Chen LL, Yang L. Complementary sequence-mediated exon circularization. Cell. 2014;159:134-47.

67. Wu HJ, Ma YK, Chen T, Wang M, Wang XJ. PsRobot: a web-based plant small RNA meta-analysis toolbox. Nucleic Acids Res. 2012;40:W22-8.

68. Shannon P, Markiel A, Ozier O, Baliga NS, Wang JT, Ramage D, Amin N, Schwikowski B, Ideker T. Cytoscape: a software environment for integrated models of biomolecular interaction networks. Genome Res. 2003;13:2498-504.

\section{Publisher's Note}

Springer Nature remains neutral with regard to jurisdictional claims in published maps and institutional affiliations.

Ready to submit your research? Choose BMC and benefit from:

- fast, convenient online submission

- thorough peer review by experienced researchers in your field

- rapid publication on acceptance

- support for research data, including large and complex data types

- gold Open Access which fosters wider collaboration and increased citations

- maximum visibility for your research: over $100 \mathrm{M}$ website views per year

At BMC, research is always in progress.

Learn more biomedcentral.com/submissions 